\title{
IDEMPOTENT STATES ON LOCALLY COMPACT QUANTUM GROUPS
}

\author{
PEKKA SALMI AND ADAM SKALSKI
}

\begin{abstract}
Idempotent states on a unimodular coamenable locally compact quantum group A are shown to be in one-to-one correspondence with right invariant expected $C^{*}$-subalgebras of A. Haar idempotents, that is, idempotent states arising as Haar states on compact quantum subgroups of $A$, are characterised and shown to be invariant under the natural action of the modular element. This leads to the one-to-one correspondence between Haar idempotents on $\mathrm{A}$ and right invariant symmetric expected $C^{*}$-subalgebras of A without the unimodularity assumption. Finally the tools developed in the first part of the paper are applied to show that the coproduct of a coamenable locally compact quantum group restricts to a continuous coaction on each right invariant expected $C^{*}$-subalgebra.
\end{abstract}

Idempotent probability measures on locally compact groups arise naturally as limit distributions of random walks. By analogy, when one considers quantum random walks in the setup provided by topological quantum groups $\left[\mathrm{FS}_{1}\right]$, one is led to consider idempotent states on locally compact quantum groups. Since the work of Kawada and Itô [KaI], idempotent probability measures have been well understood, as they all arise as Haar measures on compact subgroups. In the quantum world, as shown in [Pal], the situation is more complicated, as already some finite quantum groups admit idempotent states which cannot be canonically associated with any quantum subgroup. Motivated by this discovery U. Franz and the second-named author, later joined by R. Tomatsu, have begun a systematic investigation of idempotent states on finite and compact quantum groups $\left[\mathrm{FS}_{2-3}\right.$, FST]. In particular, necessary and sufficient conditions for such states to be Haar idempotents, i.e. to arise as Haar states on closed quantum subgroups, have been identified, and close relations to expected right invariant $C^{*}$-subalgebras (called in $\left[\mathrm{FS}_{3}\right]$ coidalgebras) uncovered. On the other hand the first-named author, inspired by the harmonic analysis considerations due to Lau and Losert showed in a recent paper [Sa] a one-to-one correspondence between compact quantum subgroups of a coamenable locally compact quantum group A and certain right invariant unital $C^{*}$-subalgebras of A.

Motivated by these developments, in this paper we study idempotent states and related structures on coamenable locally compact quantum groups in the sense of $[\mathrm{KuV}]$. At first it might appear that the algebraic formalism here is similar to that encountered in $\left[\mathrm{FS}_{2-3}\right]$ and [FST], but technical aspects of the locally compact theory (such as the absence of a natural dense Hopf *-algebra) make the problems we

2000 Mathematics Subject Classification. Primary 46L65, Secondary 43A05, 46L30, 60B15.

Key words and phrases. Locally compact quantum groups, idempotent states, compact quantum subgroups. 
investigate more complicated and necessitate developing new approaches. In particular we discover an unexpected role played by unimodularity: our proof of the main result characterising idempotent states in terms of right invariant expected $C^{*}$-subalgebras requires the assumption that the left and right Haar weights coincide. For Haar idempotents this restriction is not necessary, as we show that they are invariant in a natural sense under the modular element allowing the passage between the left and right Haar weights.

Classically it is well known that if $G$ is a locally compact group and $H$ is a compact subgroup of $G$, then $G$ acts continuously (by multiplication) on the algebra of continuous functions on $G$ constant on the cosets of $H$. Using the results obtained in the first part of the paper we show a broad quantum counterpart of this fact - a coamenable locally compact quantum group coacts continuously not only on the fixed point algebras for the canonical actions of its compact quantum subgroups (which has been earlier proved in [Sol]), but also on arbitrary right invariant expected $C^{*}$-subalgebras.

As we are interested in quantum counterparts of all regular Borel measures on a locally compact group $G$ (and not only those which are absolutely continuous with respect to the Haar measures), it is natural to work with the $C^{*}$-algebraic versions of locally compact quantum groups. The standing assumption of coamenability allows us to avoid certain technical subtleties related to the notion of a quantum subgroup and can be explained by the desire to model the classical fact that there is a natural one-to-one correspondence between closed subgroups of a locally compact group $G$ and measures on $G$ induced by Haar measures on these subgroups - in general, Haar weights on non-coamenable locally compact quantum groups need not be faithful.

The detailed plan of the paper is as follows: in Section 1 we briefly recall basic facts on weights, conditional expectations for $C^{*}$-algebras, multiplier algebras and strict topology, establish the notation and terminology related to locally compact quantum groups and prove a few technical results required later in the paper. Section 2 is devoted to idempotent states on a locally compact quantum group - in particular we show that the idempotent property of a state can be characterised by the fact that its associated convolution operator is a conditional expectation, and we introduce a natural partial order on the set of idempotent states. Section 3 contains the main results of the paper. We first establish the natural correspondence between idempotent states and right invariant expected $C^{*}$-subalgebras under the assumption of unimodularity, then prove that Haar idempotents are in a natural sense invariant under the modular element and finally combine these facts to obtain the natural correspondence between Haar idempotents and right invariant symmetric expected $C^{*}$-subalgebras in the general case. The last short section is devoted to showing that the coproduct of a coamenable locally compact quantum group restricts to a continuous coaction on each right invariant expected $C^{*}$-subalgebra.

\section{Preliminary facts}

In this section we describe basic facts related to weight-preserving conditional expectations in the $C^{*}$-algebraic context and recall the basic definitions and facts of the theory of locally compact quantum groups. The symbol $\otimes$ denotes the minimal/spatial tensor product of $C^{*}$-algebras. 
Weights and conditional expectations on $C^{*}$-algebras. We begin by gathering a few facts which are standard for normal weights on von Neumann algebras and are probably well known also in this, $C^{*}$-algebraic, context.

Definition 1.1. A weight on a $C^{*}$-algebra $\mathrm{A}$ is a map $\psi: \mathrm{A}_{+} \rightarrow[0, \infty]$ that is additive and homogenous with respect to scalars in $\mathbb{R}_{+}$. A weight $\psi$ is densely defined if

$$
\mathfrak{M}_{\psi}^{+}:=\left\{a \in \mathrm{A}_{+}: \psi(a)<\infty\right\}
$$

is dense in $\mathbf{A}_{+}$. It is faithful if the equality $\psi(a)=0$, with $a \in \mathrm{A}_{+}$, implies that $a=0$.

A weight $\psi$ on A can be uniquely extended (as a linear functional) to the linear span of $\mathfrak{M}_{\psi}^{+}$; the latter is equal to $\mathfrak{N}_{\psi}^{*} \mathfrak{N}_{\psi}$, where

$$
\mathfrak{N}_{\psi}=\left\{a \in \mathrm{A}: a^{*} a \in \mathfrak{M}_{\psi}^{+}\right\} .
$$

This can be found for example in [Tak].

Definition 1.2. Let $\psi$ be a weight on a $C^{*}$-algebra $\mathrm{A}$, and let $\mathrm{C}$ be a $C^{*}$-subalgebra of $A$. A norm-one projection $E_{C}$ from $A$ onto $C$ is called a conditional expectation. We say that $E_{\mathrm{C}}$ preserves $\psi$ if for all $a \in \mathfrak{M}_{\psi}^{+}$

$$
E_{\mathrm{C}}(a) \in \mathfrak{M}_{\psi}^{+}, \quad \psi\left(E_{\mathrm{C}}(a)\right)=\psi(a) .
$$

If a conditional expectation onto $\mathrm{C}$ preserving $\psi$ exists, $\mathrm{C}$ is called $\psi$-expected.

Note that if $E_{\mathrm{C}}$ preserves $\psi$, then by linearity the equality $\psi\left(E_{\mathrm{C}}(a)\right)=\psi(a)$ is valid for all $a \in \mathfrak{N}_{\psi}^{*} \mathfrak{N}_{\psi}$.

Lemma 1.3. Let $\psi$ be a densely defined faithful weight on a $C^{*}$-algebra A. Suppose that $\mathrm{C}$ is a $C^{*}$-subalgebra of $\mathrm{A}$ such that there exists a $\psi$-preserving conditional expectation $E_{\mathrm{C}}$ from $\mathrm{A}$ onto $\mathrm{C}$. Then $E_{\mathrm{C}}$ is uniquely determined, $E_{\mathrm{C}}\left(\mathfrak{N}_{\psi}\right) \subset \mathfrak{N}_{\psi}$ and $\mathfrak{N}_{\psi} \cap \mathrm{C}$ is dense in $\mathrm{C}$. Moreover, if we define $\mathrm{C}^{\perp}:=\left\{a \in \mathrm{A} \cap \mathfrak{N}_{\psi}: E_{\mathrm{C}}(a)=0\right\}$, then

$$
\mathrm{C}^{\perp}=\left\{a \in \mathrm{A} \cap \mathfrak{N}_{\psi}: \psi\left(c^{*} a\right)=0 \text { for all } c \in \mathrm{C} \cap \mathfrak{N}_{\psi}\right\} .
$$

Proof. Let $a \in \mathfrak{N}_{\psi}$. As a conditional expectation, $E_{\mathrm{C}}$ is a contractive completely positive map and by the Kadison-Schwarz inequality $E_{\mathrm{C}}\left(a^{*}\right) E_{\mathrm{C}}(a) \leq E_{\mathrm{C}}\left(a^{*} a\right)$. Since $a^{*} a \in \mathfrak{M}_{\psi}^{+}$and $E_{\mathrm{C}}$ is $\psi$-preserving, we have

$$
\psi\left(E_{\mathrm{C}}\left(a^{*}\right) E_{\mathrm{C}}(a)\right) \leq \psi\left(E_{\mathrm{C}}\left(a^{*} a\right)\right)=\psi\left(a^{*} a\right)<\infty .
$$

Thus $E_{\mathrm{C}}\left(\mathfrak{N}_{\psi}\right)=\mathrm{C} \cap \mathfrak{N}_{\psi}$ and $\mathbf{C} \cap \mathfrak{N}_{\psi}$ is dense in C. Further if $a \in \mathfrak{N}_{\psi}$ and $c \in \mathrm{C} \cap \mathfrak{N}_{\psi}$, then

$$
\psi\left(c^{*} a\right)=\psi\left(E_{\mathrm{C}}\left(c^{*} a\right)\right)=\psi\left(c^{*} E_{\mathrm{C}}(a)\right) .
$$

Hence if $E_{\mathrm{C}}(a)=0$, then $\psi\left(c^{*} a\right)=0$. On the other hand if $\psi\left(c^{*} a\right)=0$ for all $c \in \mathrm{C} \cap \mathfrak{N}_{\psi}$, then $0=\psi\left(E_{\mathrm{C}}(a)^{*} a\right)=\psi\left(E_{\mathrm{C}}\left(E_{\mathrm{C}}(a)^{*} a\right)\right)=\psi\left(E_{\mathrm{C}}(a)^{*} E_{\mathrm{C}}(a)\right)$ and faithfulness of $\psi$ implies that $E_{\mathrm{C}}(a)=0$.

The above arguments imply that $\mathfrak{N}_{\psi}=\mathrm{C}^{\perp} \oplus\left(\mathbf{C} \cap \mathfrak{N}_{\psi}\right)$ (as a vector space). As $\mathfrak{N}_{\psi}$ is dense in $\mathrm{A}$ and $\mathrm{C}^{\perp}$ depends only on $\mathrm{C}$ and $\psi$, the uniqueness of the $\psi$-preserving conditional expectation onto $\mathrm{C}$ follows. 
Multiplier algebra. The multiplier algebra $M(\mathrm{~A})$ of a $C^{*}$-algebra $\mathrm{A}$ is the largest unital $C^{*}$-algebra that contains $\mathrm{A}$ as an essential ideal; in other words $M(\mathrm{~A})$ is the largest reasonable unitisation of $\mathrm{A}$. We will write $1_{\mathrm{A}}$ to denote the unit of $M(\mathrm{~A})$. The strict topology on $M(\mathrm{~A})$ is the topology generated by the seminorms $x \mapsto\|x a\|$, $x \mapsto\|a x\|$, where $a$ runs through the elements of $\mathrm{A}$. $\mathrm{A} *$-homomorphism $\pi: \mathrm{A} \rightarrow$ $M(\mathrm{~B})$, where $\mathrm{B}$ is another $C^{*}$-algebra, is nondegenerate if $\pi(\mathrm{A}) \mathrm{B}$ is dense in $\mathrm{B} . \mathrm{A}^{*}$ homomorphism $\pi$ is nondegenerate if and only if the net $\left(\pi\left(e_{i}\right)\right)_{i \in I}$ converges strictly to $1_{\mathrm{B}}$ for any (or for every) bounded approximate identity $\left(e_{i}\right)_{i \in I}$ of A. Moreover, we say that a $C^{*}$-subalgebra $\mathrm{C}$ of $\mathrm{A}$ is nondegerate if the embedding $\mathrm{C} \hookrightarrow \mathrm{A}$ is nondegenerate. A bounded linear map $\mathrm{A} \rightarrow M(\mathrm{~B})$ that admits a (unique) extension to $M(\mathrm{~A})$ which is strictly continuous on bounded sets is said to be strict (this unique extension is denoted by the same symbol as the original map, unless stated otherwise). Every nondegenerate ${ }^{*}$-homomorphism is strict. So are all bounded linear functionals $\omega: A \rightarrow \mathbb{C}$ as well as slice maps of bounded linear functionals: for example $\omega \otimes \operatorname{id}_{B}: A \otimes B \rightarrow B$. Also nondegenerate completely positive maps are strict: a completely positive map $P: \mathrm{A} \rightarrow M(\mathrm{~B})$ is nondegenerate if for some bounded approximate identity $\left(e_{i}\right)_{i \in I}$ of $\mathrm{A}$ the net $\left(P\left(e_{i}\right)\right)_{i \in I}$ converges strictly to $1_{\mathrm{B}}$ in $M(\mathrm{~B})$. The last fact implies that if $\mathrm{B}$ is a nondegenerate $C^{*}$-subalgebra of $\mathrm{A}$ and $E$ is a conditional expectation from $A$ to $B$, then $E$ is strict. A good reference to nondegenerate completely positive maps, as well as multiplier algebras in general, is [Lan].

Lemma 1.4. Let $\mathrm{A}$ be a $C^{*}$-algebra, let $\mathrm{C}$ be a nondegenerate $C^{*}$-subalgebra of $\mathrm{A}$ and let $E: \mathrm{A} \rightarrow \mathrm{C}$ be a conditional expectation onto $\mathrm{C}$. Then the strict extension $\tilde{E}$ of $E$ to $M(\mathrm{~A})$ is a conditional expectation onto $M(\mathrm{C})$. In particular,

$$
\tilde{E}(m n)=\tilde{E}(m) n \quad \text { and } \quad \tilde{E}(n m)=n \tilde{E}(m)
$$

for every $m \in M(\mathrm{~A})$ and $n \in M(\mathrm{C})$.

Proof. As $\mathrm{C}$ is assumed to be nondegenerate in A, it follows from Proposition 2.3 of [Lan] that we can identify $M(\mathrm{C})$ in a canonical way with a subalgebra of $M(\mathrm{~A})$. The well-known module property of conditional expectations states that for all $a \in \mathrm{A}$, $c \in \mathrm{C}$

$$
E(a c)=E(a) c .
$$

Fix $m \in M(\mathrm{~A})$. By a version of the Kaplansky theorem for strictly dense algebras (Proposition 1.4 of [Lan]), there is a bounded net $\left(a_{i}\right)_{i \in I}$ in A that converges strictly to $m$. As $\tilde{E}$ is strictly continuous on bounded sets, we obtain

$$
E(m c)=\lim _{i \in I} E\left(a_{i} c\right)=\lim _{i \in I} E\left(a_{i}\right) c=\tilde{E}(m) c .
$$

Let then $n \in M(\mathrm{C}) \subset M(\mathrm{~A})$ and let $\left(c_{i}\right)_{i \in I}$ be a bounded net in $\mathrm{C}$ strictly convergent to $n$. By nondegeneracy it is also strictly convergent to $n$ in $M(\mathrm{~A})$. Hence $\left(m c_{i}\right)_{i \in I}$ is a bounded net in A strictly convergent to $m n$, and

$$
\tilde{E}(m n)=\lim _{i \in I} E\left(m c_{i}\right)=\lim _{i \in I} \tilde{E}(m) c_{i}=\tilde{E}(m) n .
$$

In particular,

$$
\tilde{E}(n)=\tilde{E}\left(1_{c} n\right)=\tilde{E}\left(1_{\mathrm{c}}\right) n=n,
$$

so $\tilde{E}$ maps $M(\mathrm{~A})$ onto $M(\mathrm{C})$. Since $\tilde{E}$ is also a projection of norm one, it is a conditional expectation. 
Elements affiliated with a $C^{*}$-algebra. The noncommutative analogue of unbounded continuous functions is given by elements affiliated with a $C^{*}$-algebra. These were introduced by Woronowicz in $\left[\mathrm{Wo}_{1}\right]$ (and also by Baaj in $\left[\mathrm{Ba}_{1}\right]$ ). Let $\mathrm{A}$ be a $C^{*}$-algebra. A densely defined operator $T: \mathrm{A} \supset \operatorname{dom}(T) \rightarrow \mathrm{A}$ is affiliated with $\mathrm{A}$ if there exists $z_{T} \in M(\mathrm{~A})$ with $\left\|z_{T}\right\| \leq 1$ such that

$$
x \in \operatorname{dom}(T) \text { and } y=T x
$$

if and only if

$$
x=\left(1_{\mathrm{A}}-z_{T}^{*} z_{T}\right)^{1 / 2} a \text { for some } a \in \mathrm{A} \text { and } y=z_{T} a .
$$

The element $z_{T}$, which is unique when it exists, is called the $z$-transform of $T$. Elements of the multiplier algebra $M(\mathrm{~A})$ are exactly the bounded operators on $\mathrm{A}$ that are affiliated with $A$. If $A$ is unital, every element affiliated with $A$ is in $A$. If $\mathrm{A} \approx C_{0}(X)$ for a locally compact space $X$, then the elements affiliated with $\mathrm{A}$ correspond precisely to the continuous, not necessarily bounded, functions on $X$.

Suppose that $\mathrm{A}$ and $\mathrm{B}$ are $C^{*}$-algebras, $\pi: \mathrm{A} \rightarrow M(\mathrm{~B})$ is a nondegenerate ${ }^{*}$ homomorphism, and $T$ is affiliated with $\mathrm{A}$. By Theorem 1.2 of $\left[\mathrm{Wo}_{1}\right]$, there is a unique operator $\pi(T)$ such that $\pi(T)$ is affiliated with $\mathrm{B}$, the set $\pi(\operatorname{dom}(T)) \mathrm{B}$ is a core of $\pi(T)$ and

$$
\pi(T)(\pi(a) b)=\pi(T a) b
$$

for every $a$ in $\operatorname{dom}(T)$ and $b$ in B. Moreover, the composition rule

$$
\rho(\pi(T))=(\rho \circ \pi)(T)
$$

is true when both $\pi$ and $\rho$ are nondegenerate ${ }^{*}$-homomorphisms.

Woronowicz $\left[\mathrm{Wo}_{1}\right]$ developed functional calculus of normal affiliated elements; see also $[\mathrm{NaW}]$ and $\left[\mathrm{Ku}_{2}\right]$. We will need it to deal with powers of strictly positive elements. An operator $T$ affiliated with $\mathrm{A}$ is strictly positive if it is positive (i.e. $z_{T}$ is positive) and has a dense range. Such an operator is necessarily injective. If $T$ is a normal element affiliated with $\mathrm{A}$ and $f$ is a continuous complex-valued function defined on the spectrum of $T$, then $f(T)$ is a well-defined operator that is affiliated with $\mathrm{A}$. Moreover, if $\pi: \mathrm{A} \rightarrow M(\mathrm{~B})$ is a nondegenerate ${ }^{*}$-homomorphism, then

$$
f(\pi(T))=\pi(f(T)) .
$$

This is equation (1.4) of [NaW]. However, we need to apply the above equation also to negative powers of strictly positive operators in which case we need to exclude 0 from the spectrum of $T$. The equation still holds true in that case, as shown in Proposition 6.17 of $\left[\mathrm{Ku}_{2}\right]$. By Proposition 7.11 of $\left[\mathrm{Ku}_{2}\right]$, all real powers of a strictly positive operator affiliated with $A$ are also strictly positive and affiliated with $A$.

Locally compact quantum groups in the $C^{*}$-algebraic framework. We follow the $C^{*}$-algebraic approach to locally compact quantum groups due to Kustermans and Vaes $[\mathrm{KuV}]$.

Definition 1.5. A coproduct on a $C^{*}$-algebra $\mathrm{A}$ is a nondegenerate *-homomorphism $\Delta: \mathrm{A} \rightarrow M(\mathrm{~A} \otimes \mathrm{A})$ that is coassociative:

$$
\left(\operatorname{id}_{\mathrm{A}} \otimes \Delta\right) \Delta=\left(\Delta \otimes \operatorname{id}_{\mathrm{A}}\right) \Delta .
$$

A $C^{*}$-algebra $\mathrm{A}$ with a coproduct $\Delta$ is a locally compact quantum group if the quantum cancellation laws

$$
\overline{\operatorname{span}} \Delta(\mathrm{A})\left(\mathrm{A} \otimes 1_{\mathrm{A}}\right)=\mathrm{A} \otimes \mathrm{A}, \quad \overline{\operatorname{span}} \Delta(\mathrm{A})\left(1_{\mathrm{A}} \otimes \mathrm{A}\right)=\mathrm{A} \otimes \mathrm{A}
$$


are satisfied and if there exist faithful KMS-weights $\phi$ and $\psi$ on A such that $\phi$ is left invariant

$$
\phi((\omega \otimes \mathrm{id}) \Delta(a))=\omega\left(1_{\mathrm{A}}\right) \phi(a) \quad\left(\omega \in \mathrm{A}_{+}^{*}, a \in \mathfrak{M}_{\phi}^{+}\right)
$$

and $\psi$ is right invariant

$$
\psi((\mathrm{id} \otimes \omega) \Delta(a))=\omega\left(1_{\mathrm{A}}\right) \psi(a) \quad\left(\omega \in \mathrm{A}_{+}^{*}, a \in \mathfrak{M}_{\psi}^{+}\right) .
$$

(The KMS-condition is a technical condition which we shall not use explicitly. For our purposes it is enough to know that the weights are densely defined and lower semicontinuous.) The left invariant weight $\phi$ is called the left Haar weight and the right invariant $\psi$ the right Haar weight. The quantum group A is said to be unimodular if $\phi=\psi$. The faithfulness assumption means that we are dealing with the reduced (as opposed to the universal, see $\left[\mathrm{Ku}_{3}\right]$ ) version of $\mathrm{A}$.

If the $C^{*}$-algebra $\mathrm{A}$ is unital, we say that the quantum group $\mathrm{A}$ is compact. In this case there is a unique Haar state of $\mathrm{A}$ that is both left and right invariant. Compact quantum groups are often defined so that the Haar state is not necessarily faithful. This makes no difference in our setting, where we consider compact quantum subgroups of a coamenable locally compact quantum group: see the remark at the end of this section.

A counit is a linear functional $\epsilon$ on $A$ such that

$$
\left(\epsilon \otimes \operatorname{id}_{\mathrm{A}}\right) \Delta=\left(\operatorname{id}_{\mathrm{A}} \otimes \epsilon\right) \Delta=\operatorname{id}_{\mathrm{A}} .
$$

A quantum group that has a bounded counit is said to be coamenable [BeT].

Suppose that A is a locally compact quantum group. Then the GNS representation associated with the left Haar weight $\phi$ is faithful, and so we may consider A as a $C^{*}$-subalgebra of $B(H)$, where $H$ is the Hilbert space obtained from the GNS construction.

The right multiplicative unitary $V$ is a very special unitary operator on $H \otimes H$ which belongs to the multiplier algebra $M(K(H) \otimes \mathrm{A})$ ) (where $K(H)$ denotes the algebra of compact operators on $H$ ) and which completely determines A. The coproduct $\Delta$ is given by

$$
\Delta(a)=V(a \otimes 1) V^{*} \quad(a \in \mathrm{A})
$$

and the $C^{*}$-algebra $\mathrm{A}$ itself is the norm closure of

$$
\left\{(\sigma \otimes \mathrm{id}) V: \sigma \in B(H)_{*}\right\} .
$$

Here $B(H)_{*}$ denotes the predual of $B(H)$ : the weak*-continuous functionals on $B(H)$. The multiplicative unitary $V$ satisfies the pentagonal relation

$$
V_{12} V_{13} V_{23}=V_{23} V_{12} \text {. }
$$

In the above formula we use the leg numbering notation: $V_{12}=V \otimes 1, V_{23}=1 \otimes V$ and $V_{13}=(1 \otimes \Sigma)(V \otimes 1)(1 \otimes \Sigma), \Sigma(\xi \otimes \eta)=\eta \otimes \xi$.

The antipode $S$ of the quantum group $\mathrm{A}$ is a closed, densely defined operator on A. The elements $(\sigma \otimes \mathrm{id}) V$, with $\sigma \in B(H)_{*}$, form a core of $S$ and

$$
S((\sigma \otimes \mathrm{id}) V)=(\sigma \otimes \mathrm{id}) V^{*} .
$$

It is more common to use the formula

$$
S((\mathrm{id} \otimes \sigma) W)=(\mathrm{id} \otimes \sigma) W^{*},
$$


where $W$ is the left multiplicative unitary; see Proposition 8.3 of $[\mathrm{KuV}]$. Equation (1.3) follows from Proposition 8.3 of $[\mathrm{KuV}]$ by using the identity

$$
V=(\hat{J} \otimes \hat{J}) \Sigma W^{*} \Sigma(\hat{J} \otimes \hat{J})
$$

where $\Sigma: H \otimes H \rightarrow H \otimes H$ is the flip map and $\hat{J}: H \rightarrow H$ is the anti-unitary modular conjugation coming from the Tomita-Takesaki theory for the left Haar weight of the dual locally compact quantum group $\hat{A}$ (see $\left[\mathrm{Va}_{1}\right]$ ).

When $\mathrm{A}$ is not unimodular, the modular element $\delta$ provides a passage between the Haar weights $\phi$ and $\psi$. The modular element $\delta$ is a strictly positive, unbounded operator on $H$ that is affiliated with the $C^{*}$-algebra A. Formally,

$$
\psi(a)=\phi\left(\delta^{1 / 2} a \delta^{1 / 2}\right), \quad a \in \mathfrak{M}_{\psi}^{+} .
$$

A $C^{*}$-algebra $\mathrm{B}$ acts in a natural way on $\mathrm{B}^{*}$ : for each $\mu \in \mathrm{B}^{*}, b \in \mathrm{B}$ we define $\mu_{b} \in \mathrm{B}^{*}$ and ${ }_{b} \mu \in \mathrm{B}^{*}$ by

$$
\mu_{b}(c)=\mu(b c), \quad{ }_{b} \mu(c)=\mu(c b) .
$$

Let $\mathrm{A}$ be a coamenable locally compact quantum group with left and right invariant weights $\phi$ and $\psi$, respectively. If $\mu, \nu \in \mathrm{A}^{*}$ we write

$$
\mu \star \nu=(\mu \otimes \nu) \Delta, \quad L_{\mu}=\left(\mu \otimes \operatorname{id}_{\mathrm{A}}\right) \Delta, \quad R_{\mu}=\left(\operatorname{id}_{\mathrm{A}} \otimes \mu\right) \Delta .
$$

The fact that the maps $L_{\mu}$ and $R_{\mu}$ take values in $\mathrm{A}$ (and not just in $M(\mathrm{~A})$ ) is a consequence of the inclusions of both $\Delta(\mathrm{A})\left(\mathrm{A} \otimes 1_{\mathrm{A}}\right)$ and $\Delta(\mathrm{A})\left(1_{\mathrm{A}} \otimes \mathrm{A}\right)$ in $\mathrm{A} \otimes \mathrm{A}$ and the usual factorisation of functionals on $A$ resulting from Cohen's factorisation theorem (for all $\mu \in \mathrm{A}^{*}$ there exists $\nu, \nu^{\prime} \in \mathrm{A}^{*}$ and $a, a^{\prime} \in \mathrm{A}$ such that $\mu=\nu_{a}={ }_{a^{\prime}} \nu^{\prime}$ ). It can be easily checked that if $\mu, \nu \in \mathrm{A}^{*}$ then $L_{\mu}=L_{\nu}$ implies $\mu=\nu$ and we always have $L_{\mu} L_{\nu}=L_{\nu \star \mu}$. The following lemma gives a useful characterisation for the maps of the form $L_{\omega}$; it is closely related to Theorem 2.4 in [LiS].

Lemma 1.6. Let $\mathrm{A}$ be a coamenable locally compact quantum group and let $T$ : $\mathrm{A} \rightarrow \mathrm{A}$ be a completely bounded map such that $T \otimes \mathrm{id}_{\mathrm{A}}: \mathrm{A} \otimes \mathrm{A} \rightarrow \mathrm{A} \otimes \mathrm{A}$ is strictly continuous on bounded subsets. Suppose that $G \subset \mathrm{A}^{*}$ is weak*-dense and invariant under the right action of some dense subalgebra $\mathcal{A}$ of $\mathrm{A}$. Then the following conditions are equivalent:

(i) $T=L_{\mu}$, for some $\mu \in \mathrm{A}^{*}$;

(ii) $\left(T \otimes \operatorname{id}_{\mathrm{A}}\right) \Delta=\Delta T$;

(iii) $T R_{\nu}=R_{\nu} T$ for all $\nu \in G$.

If the above conditions hold, $T=R_{\epsilon \circ T}$ is a linear combination of completely positive nondegenerate maps and the equality in (iii) is valid for all $\nu \in \mathrm{A}^{*}$.

Proof. (i) $\Rightarrow$ (ii) Standard calculation.

(ii) $\Rightarrow$ (i) Application of $\epsilon \otimes \mathrm{id}_{\mathrm{A}}$ to the equality in (ii).

(ii) $\Rightarrow$ (iii) Fix $\nu \in \mathrm{A}^{*}$ and apply id $\mathrm{id}_{\mathrm{A}} \otimes \nu$ to the equality in (ii).

(iii) $\Rightarrow$ (ii) Let $\nu \in G$ and $a \in \mathcal{A}$. As $\nu_{a} \in G$ we deduce from (iii) that for all $x \in \mathrm{A}$

$$
\left(\operatorname{id}_{\mathrm{A}} \otimes \nu\right)\left(\left(T \otimes \operatorname{id}_{\mathrm{A}}\right)\left(\left(1_{\mathrm{A}} \otimes a\right) \Delta(x)\right)\right)=\left(\operatorname{id}_{\mathrm{A}} \otimes \nu\right)\left(\left(1_{\mathrm{A}} \otimes a\right) \Delta(T(x))\right) .
$$

As the family $\left\{\mu \otimes \nu: \mu \in \mathrm{A}^{*}, \nu \in G\right\}$ separates points in $\mathrm{A} \otimes \mathrm{A}$, the last formula is equivalent to

$$
\left(T \otimes \operatorname{id}_{\mathrm{A}}\right)\left(\left(1_{\mathrm{A}} \otimes a\right) \Delta(x)\right)=\left(1_{\mathrm{A}} \otimes a\right) \Delta(T(x))
$$


holding for all $a \in \mathcal{A}, x \in \mathrm{A}$. That this implies (ii) is a consequence of density of $\mathcal{A}$ in $\mathrm{A}$ and the definition of the multiplier algebra.

A compact quantum subgroup of $A$ is a compact quantum group $B$ with a surjective ${ }^{*}$-homomorphism $\pi_{\mathrm{B}}: \mathrm{A} \rightarrow \mathrm{B}$ such that $\left(\pi_{\mathrm{B}} \otimes \pi_{\mathrm{B}}\right) \Delta_{\mathrm{A}}=\Delta_{\mathrm{B}} \pi_{\mathrm{B}}$, where $\Delta_{\mathrm{A}}$ and $\Delta_{B}$ denote the coproducts of $A$ and $B$, respectively. Although the morphism $\pi_{B}$ is an essential part of the definition, we shall usually suppress it and say that $B$ is a compact quantum subgroup of $A$.

Woronowicz defined compact quantum group as a unital $C^{*}$-algebra with a coproduct satisfying the quantum cancellation laws and showed that such a compact quantum group has always a unique Haar state which, however, does not need to be faithful. Although following the framework of $[\mathrm{KuV}]$ we defined compact quantum group more restrictively, our setting actually covers also the more general situation. Indeed, suppose that we have a coamenable locally compact quantum group A which has a Woronowicz-type compact quantum subgroup B. Then we can quotient out the kernel of the Haar state of $B$ to obtain a compact quantum subgroup $B^{\prime}$ of $A$ in our sense. By Theorem 8 of $[\mathrm{Sa}]$ a compact quantum subgroup of a coamenable locally compact quantum group is always coamenable, and it follows that $B=B^{\prime}$.

\section{Definition AND BASIC PROPERTIES OF IDEMPotent STATES ON LOCALLY COMPACT QUANTUM GROUPS.}

From now on we assume that $A$ is a coamenable locally compact quantum group. The following definition is central to the paper.

Definition 2.1. A state $\omega \in \mathrm{A}^{*}$ is called an idempotent state if $\omega \star \omega=\omega$.

As stated in the introduction, idempotent states on locally compact quantum groups can arise from Haar states of compact quantum subgroups. This motivates the next definition.

Definition 2.2. We call a state $\omega \in \mathrm{A}^{*}$ a Haar idempotent if there exists a compact quantum subgroup $\mathrm{B}$ of $\mathrm{A}$ such that $\omega=h_{\mathrm{B}} \circ \pi_{\mathrm{B}}$ where $h_{\mathrm{B}}$ is the Haar state of $\mathrm{B}$ and $\pi_{\mathrm{B}}: \mathrm{A} \rightarrow \mathrm{B}$ is the surjective morphism associated with the quantum subgroup B.

Each Haar idempotent is an idempotent state; in the next section we will provide a characterisation of the Haar property in terms of various objects associated with a given idempotent state.

It is easy to see (see the beginning of the proof of Theorem 2.4 below) that $\omega \in \mathrm{A}^{*}$ is an idempotent state if and only if $L_{\omega}$ (or, equivalently, $R_{\omega}$ ) is a unital positive idempotent map. It is tempting to replace the last statement by saying that $L_{\omega}$ is a conditional expectation. To show that it is indeed possible we need some preparations.

The next lemma was proved for compact quantum groups in $\left[\mathrm{FS}_{3}\right]$ (Lemma 3.1 there). As can be seen from its proof in the expanded arXiv version of that paper, apart from the Cauchy-Schwarz inequality for states the only other ingredient used is that the linear spans of $\Delta(A)\left(A \otimes 1_{A}\right)$ and $\Delta(A)\left(1_{A} \otimes A\right)$ are dense in $A \otimes A$. Since this remains true when $A$ is a locally compact quantum group, and all algebraic manipulations can be justified via strictness arguments, we obtain the following result. 
Lemma 2.3. Let $\sigma$ and $\omega$ be states on A and assume that $\sigma \star \omega=\omega \star \sigma=\omega$. Let $b \in \mathrm{A}$. Then

$$
\omega \star_{b} \sigma=\sigma(b) \omega .
$$

In particular, if $\omega \in \mathrm{A}^{*}$ is an idempotent state, then $\omega \star_{b} \omega=\omega(b) \omega$.

Theorem 2.4. A functional $\omega \in \mathrm{A}^{*}$ is an idempotent state if and only if $L_{\omega}$ (or, equivalently, $\left.R_{\omega}\right)$ is a conditional expectation.

Proof. Assume first that $L_{\omega}$ is a conditional expectation. Then it is automatically completely positive, so that $\omega=\epsilon \circ L_{\omega}$ is a contractive positive functional. As $L_{\omega}=L_{\omega} L_{\omega}=L_{\omega \star \omega}$, we obtain that $\omega=\omega \star \omega$. The strict extension of this formula to $M(\mathrm{~A})$ shows that $\omega\left(1_{\mathrm{A}}\right)=\omega\left(1_{\mathrm{A}}\right)^{2}$. As $L_{\omega}$, so also $\omega$, cannot be equal to 0 , we must have $\omega\left(1_{\mathrm{A}}\right)=1$ and $\omega$ is an idempotent state.

Assume now that $\omega$ is an idempotent state. Then $L_{\omega}: \mathrm{A} \rightarrow \mathrm{A}$ is a completely positive nondegenerate idempotent map. Complete positivity follows from the fact that both slice maps with positive functionals and *-homomorphisms are completely positive (see for example [ $\left.\mathrm{Wa}_{2}, \mathrm{p} .4\right]$ ); nondegeneracy is a consequence of the fact that $\omega$ is a state. To show that $L_{\omega}$ is a conditional expectation it is enough to check that its image is an algebra. To that end it suffices to show that for all $a, b \in \mathrm{A}$

$$
L_{\omega}(a) L_{\omega}(b)=L_{\omega}\left(L_{\omega}(a) b\right) .
$$

Let $a, b, c, d \in \mathrm{A}$. Write $\Delta_{2}: \mathrm{A} \rightarrow M(\mathrm{~A} \otimes \mathrm{A} \otimes \mathrm{A})$ for $\left(\Delta \otimes \mathrm{id}_{\mathrm{A}}\right) \Delta=\left(\mathrm{id}_{\mathrm{A}} \otimes \Delta\right) \Delta$ and compute:

$$
\begin{aligned}
\left(\omega \otimes \omega \otimes \operatorname{id}_{\mathrm{A}}\right) & \left(\Delta_{2}(a)\left(1_{\mathrm{A}} \otimes c \otimes d\right)\right)=\left(\omega \otimes{ }_{c} \omega \otimes \operatorname{id}_{\mathrm{A}}\right)\left(\Delta_{2}(a)\left(1_{\mathrm{A}} \otimes 1_{\mathrm{A}} \otimes d\right)\right) \\
= & \left(\left(\omega \star_{c} \omega\right) \otimes \operatorname{id}_{\mathrm{A}}\right)\left(\Delta(a)\left(1_{\mathrm{A}} \otimes d\right)\right)=\omega(c)\left(\omega \otimes \operatorname{id}_{\mathrm{A}}\right)\left(\Delta(a)\left(1_{\mathrm{A}} \otimes d\right)\right) \\
= & L_{\omega}(a)\left(\omega \otimes \mathrm{id}_{\mathrm{A}}\right)(c \otimes d)
\end{aligned}
$$

(note that Lemma 2.3 was used in the third equality). A simple norm limit argument shows that if $x \in \mathrm{A} \otimes \mathrm{A}$, then

$$
\left(\omega \otimes \omega \otimes \operatorname{id}_{\mathrm{A}}\right)\left(\Delta_{2}(a)\left(1_{\mathrm{A}} \otimes x\right)\right)=L_{\omega}(a)\left(\omega \otimes \operatorname{id}_{\mathrm{A}}\right)(x) .
$$

Further, using the fact that $\Delta(\mathrm{A})\left(\mathrm{A} \otimes 1_{\mathrm{A}}\right)$ is contained in $\mathrm{A} \otimes \mathrm{A}$ and all maps in the formula above are strict we deduce that for each $y \in \Delta(\mathrm{A})$

$$
\left(\omega \otimes \omega \otimes \operatorname{id}_{\mathrm{A}}\right)\left(\Delta_{2}(a)\left(1_{\mathrm{A}} \otimes y\right)\right)=L_{\omega}(a)\left(\omega \otimes \operatorname{id}_{\mathrm{A}}\right)(y)
$$

(to prove it formally we apply identity $(2.2)$ to $y\left(e_{i} \otimes 1_{\mathrm{A}}\right)$ for an approximate identity $\left(e_{i}\right)_{i \in I}$ and pass to the limit). In particular,

$$
\begin{aligned}
L_{\omega}\left(L_{\omega}(a) b\right) & =L_{\omega}\left(\left(\omega \otimes \operatorname{id}_{\mathrm{A}}\right)\left(\Delta(a)\left(1_{\mathrm{A}} \otimes b\right)\right)\right) \\
& =\left(\omega \otimes L_{\omega}\right)\left(\Delta(a)\left(1_{\mathrm{A}} \otimes b\right)\right)=\left(\omega \otimes \omega \otimes \operatorname{id}_{\mathrm{A}}\right)\left(\Delta_{2}(a)\left(1_{\mathrm{A}} \otimes \Delta(b)\right)\right) \\
& =L_{\omega}(a)\left(\omega \otimes \operatorname{id}_{\mathrm{A}}\right)(\Delta(b))=L_{\omega}(a) L_{\omega}(b) .
\end{aligned}
$$

The arguments above can be easily adapted to work with the map $R_{\omega}$.

Lemma 2.3 has other useful consequences. Recall that the multiplicative domain of a state $\omega$ of $A$ is the set

$$
\{x \in \mathrm{A}: \omega(a x)=\omega(x a)=\omega(a) \omega(x) \text { for every } a \in \mathrm{A}\} .
$$

Lemma 2.5. Let $\omega$ be an idempotent state on $\mathrm{A}$. Then $L_{\omega}(\mathrm{A})$ is contained in the multiplicative domain of $\omega$. 
Proof. Let $a \in$ A. Since $L_{\omega}$ is a conditional expectation,

$$
\omega\left(L_{\omega}(a)^{*} L_{\omega}(a)\right)=\omega\left(L_{\omega}\left(L_{\omega}\left(a^{*}\right) a\right)\right)=\omega\left(L_{\omega}\left(a^{*}\right) a\right)=\omega \star_{a} \omega\left(a^{*}\right) .
$$

By Lemma 2.3,

$$
\omega \star_{a} \omega\left(a^{*}\right)=\omega(a) \omega\left(a^{*}\right)=\omega\left(L_{\omega}(a)^{*}\right) \omega\left(L_{\omega}(a)\right) .
$$

Similarly,

$$
\omega\left(L_{\omega}(a) L_{\omega}(a)^{*}\right)=\omega\left(L_{\omega}(a)\right) \omega\left(L_{\omega}(a)^{*}\right),
$$

so Choi's theorem [Pau, Theorem 3.18], applied to the strict extension of $\omega$ to $\tilde{\omega} \in M(\mathrm{~A})^{*}$ implies that $L_{\omega}(a)$ is in the multiplicative domain of $\tilde{\omega}$, so also in the multiplicative domain of $\omega$.

In Proposition 3.4 of [FST] it is shown that idempotent states on compact quantum groups are in a sense invariant under the antipode. The next proposition shows that the same remains true in the locally compact context. The proof is different from that in $[\mathrm{FST}]$.

Proposition 2.6. Every idempotent state $\omega$ satisfies $\omega S(a)=\omega(a)$ for every a in $\operatorname{dom}(S)$.

Proof. By Theorem 2.4, $L_{\omega}$ is a conditional expectation onto $\mathrm{C}:=L_{\omega}(\mathrm{A})$. It follows that id $\otimes L_{\omega}: K(H) \otimes \mathrm{A} \rightarrow K(H) \otimes \mathrm{C}$ is also a conditional expectation. Next we check that $C$ is nondegenerate in $A$ : we have

$$
\mathrm{CA}=L_{\omega}(\mathrm{A}) \mathrm{A}=\left(\omega \otimes \mathrm{id}_{\mathrm{A}}\right)\left(\Delta(\mathrm{A})\left(1_{\mathrm{A}} \otimes \mathrm{A}\right)\right),
$$

so the quantum cancellation law $\overline{\operatorname{span}} \Delta(A)\left(1_{A} \otimes A\right)=A \otimes A$ implies that the linear span of CA is dense in A. Then Cohen's factorisation theorem implies that CA is dense in $\mathrm{A}$. Since $\mathrm{C}$ is nondegenerate in $\mathrm{A}$, it follows that $K(H) \otimes \mathrm{C}$ is nondegenerate in $K(H) \otimes \mathrm{A}$. Hence the conditional expectation id $\otimes L_{\omega}$ extends to a conditional expectation on $M(K(H) \otimes \mathrm{A})$ by Lemma 1.4.

Put $p=($ id $\otimes \omega) V$. (It turns out that $p$ is an orthogonal projection, hence the notation.) Now

$$
\left(\mathrm{id} \otimes L_{\omega}\right) V=(\mathrm{id} \otimes \omega \otimes \mathrm{id}) V_{12} V_{13}=(p \otimes 1) V .
$$

On the other hand, $p^{*}=(\mathrm{id} \otimes \omega) V^{*}$ and

$$
\left(\mathrm{id} \otimes L_{\omega}\right) V^{*}=V^{*}\left(p^{*} \otimes 1\right) .
$$

Then

$$
\begin{aligned}
p p^{*} \otimes 1_{\mathrm{A}} & =\left(p \otimes 1_{\mathrm{A}}\right) V V^{*}\left(p^{*} \otimes 1_{\mathrm{A}}\right)=\left(\left(\mathrm{id} \otimes L_{\omega}\right) V\right)\left(\left(\mathrm{id} \otimes L_{\omega}\right) V^{*}\right) \\
& =\left(\mathrm{id} \otimes L_{\omega}\right)\left(\left(\left(\mathrm{id} \otimes L_{\omega}\right) V\right) V^{*}\right)
\end{aligned}
$$

because id $\otimes L_{\omega}$ is a conditional expectation on $M(K(H) \otimes \mathrm{A})$. Continue the calculation:

$$
p p^{*} \otimes 1=\left(\mathrm{id} \otimes L_{\omega}\right)\left((p \otimes 1) V V^{*}\right)=\left(\mathrm{id} \otimes L_{\omega}\right)(p \otimes 1)=p \otimes 1 .
$$

Hence $p p^{*}=p$ and $p$ is an orthogonal projection.

Now for every $\sigma$ in $B(H)_{*}$,

$$
\omega((\sigma \otimes \mathrm{id}) V)=\sigma(p)=\sigma\left(p^{*}\right)=\omega\left((\sigma \otimes \mathrm{id}) V^{*}\right)=\omega S((\sigma \otimes \mathrm{id}) V) .
$$

Since $\left\{(\sigma \otimes \mathrm{id}) V: \sigma \in B(H)_{*}\right\}$ is a core of $S$, we have $\omega(a)=\omega S(a)$ for every $a$ in $\operatorname{dom}(S)$. 
Classically the subgroups of a given group form a lattice. The resulting partial order motivated a natural partial order $\prec$ on idempotent states on a finite quantum group introduced and studied in $\left[\mathrm{FS}_{2}\right]$. Below we show that an analogous partial ordering can be considered also for idempotent states on a locally compact quantum group.

Definition 2.7. If $\omega, \omega^{\prime} \in A^{*}$ are idempotent states, we write $\omega \prec \omega^{\prime}$ if $\omega \star \omega^{\prime}=\omega^{\prime}$

To prove that $\prec$ is antisymmetric, we need to exploit the properties of idempotent states established above.

Proposition 2.8. Suppose that $\omega$ and $\omega^{\prime}$ are idempotent states such that $\omega \prec \omega^{\prime}$, i.e. $\omega \star \omega^{\prime}=\omega^{\prime}$. Then also $\omega^{\prime} \star \omega=\omega^{\prime}$. Moreover, if we have also $\omega^{\prime} \prec \omega$, then $\omega=\omega^{\prime}$.

Proof. By Proposition 2.6, the functionals $\omega S$ and $\omega^{\prime} S$ are bounded and their bounded extensions to all of $A$ are $\omega$ and $\omega^{\prime}$, respectively. Lemma 5.25 of $[\mathrm{KuV}]$ implies that for every $a$ in $\operatorname{dom}(S)$

$$
\omega^{\prime} \star \omega(a)=\left(\omega \otimes \omega^{\prime}\right) \Delta(S(a))=\omega^{\prime} S(a)=\omega^{\prime}(a) .
$$

As for the second statement, if $\omega \prec \omega^{\prime}$, then $\omega=\omega^{\prime} \star \omega$, which by the beginning of the proof is equal to $\omega^{\prime}$.

\section{Correspondence BetWeEn IDEmpotent StATES AND Right INVARIANT EXPECTED $C^{*}$-SUBALGEBRAS}

This section contains the main results of the paper, establishing the correspondence between idempotent states and right invariant expected $C^{*}$-subalgebras (under the assumption of unimodularity) and between Haar idempotents and right invariant symmetric expected $C^{*}$-subalgebras. They extend on one hand Theorem 4.1 of $\left[\mathrm{FS}_{3}\right]$ and on the other Theorem 13 of $[\mathrm{Sa}]$. Again A denotes a coamenable locally compact quantum group.

Definition 3.1. A $C^{*}$-subalgebra $\mathrm{C} \subset \mathrm{A}$ is said to be right invariant if $R_{\mu}(\mathrm{C}) \subset \mathrm{C}$ for all $\mu \in \mathrm{A}^{*}$. It is said to be expected if there exists a conditional expectation $E_{\mathrm{C}}$ onto $\mathrm{C}$ that is both $\psi$-preserving and $\phi$-preserving.

A nondegenerate $C^{*}$-subalgebra $C$ of a coamenable quantum group $\mathrm{A}$ is right invariant if and only if $\Delta(\mathrm{C}) \subset M(\mathrm{C} \otimes \mathrm{A})$. Indeed, if $\Delta(\mathrm{C}) \subset M(\mathrm{C} \otimes \mathrm{A})$, then $R_{\mu}(\mathrm{C}) \subset M(\mathrm{C}) \cap \mathrm{A}$ for every $\mu \in \mathrm{A}^{*}$, and the nondegeneracy of $\mathrm{C}$ implies that $R_{\mu}(\mathrm{C}) \subset \mathrm{C}$. The converse is shown in Theorem 4.2 below, but we can also use the so-called slice map property to give a short argument. Suppose that $R_{\mu}(c) \in \mathrm{C}$ for every $\mu \in \mathrm{A}^{*}$ and $c \in \mathrm{C}$. Then for every $a \in \mathrm{A}, d \in \mathrm{C}$ and $\nu \in \mathrm{A}^{*}$,

$$
(d \otimes a) \Delta(c) \in \mathrm{A} \otimes \mathrm{A} \quad \text { and } \quad\left(\operatorname{id}_{\mathrm{A}} \otimes \nu\right)(d \otimes a) \Delta(c) \in \mathrm{C} \otimes \mathrm{A} .
$$

Since $\mathrm{A}$ is coamenable, it follows that $\mathrm{A}$ is a nuclear $C^{*}$-algebra [BeT] and so has the slice map property introduced by Wassermann [Wa $]$. By the slice map property, (3.1) implies that $(d \otimes a) \Delta(c) \in \mathrm{C} \otimes \mathrm{A}$. The other side can be dealt with similarly, so we see that $\Delta(c) \in M(\mathrm{C} \otimes \mathrm{A})$.

The relation between right invariant subalgebras and idempotent states can be described by the two propositions below.

Proposition 3.2. If $\omega \in \mathrm{A}^{*}$ is an idempotent state, then $L_{\omega}(\mathrm{A})$ is a right invariant $\phi$-expected $C^{*}$-subalgebra of $\mathrm{A}$. 
Proof. The statement is a direct consequence of Theorem 2.4 and the definition of the left Haar weight $\phi$.

Proposition 3.3. If $\mathrm{C}$ is a right invariant $\psi$-expected $C^{*}$-subalgebra of $\mathrm{A}$, then there exists a unique idempotent state $\omega \in \mathrm{A}^{*}$ such that $\mathrm{C}=L_{\omega}(\mathrm{A})$.

Proof. Let $E_{\mathrm{C}}$ denote the $\psi$-preserving conditional expectation onto a right invariant $C^{*}$-subalgebra $C$. We want to show that $E_{\mathrm{C}}$ is of the form $L_{\omega}(\mathrm{A})$ for an idempotent state $\omega \in \mathrm{A}^{*}$. Due to Lemma 1.6 and Theorem 2.4 it suffices to show that

$$
E_{\mathrm{C}} R_{\nu}=R_{\nu} E_{\mathrm{C}}
$$

for every $\nu \in G$, where $G$ is a weak ${ }^{*}$-dense subspace of $\mathrm{A}^{*}$ that is invariant under the action of a dense subalgebra of $\mathrm{A}$. Let $L^{1}(\mathrm{~A})$ be the collection of $\nu \in \mathrm{A}^{*}$ that are restrictions of weak*-continuous functionals on $B(H)$. Moreover, let $L_{\sharp}^{1}(\mathrm{~A})$ be the collection of all $\nu \in L^{1}(\mathrm{~A})$ for which there exists $\nu^{\sharp} \in L^{1}(\mathrm{~A})$ such that $\bar{\nu}(S(a))=\nu^{\sharp}(a)$ for every $a \in \operatorname{dom}(S)$ (here $\bar{\nu}$ is defined by $\bar{\nu}(a)=\overline{\nu\left(a^{*}\right)}$ ). Now we consider $G=L_{\sharp}^{1}(\mathrm{~A})$ which is weak ${ }^{*}$-dense in $\mathrm{A}^{*}$ and invariant under the right action of the dense subalgebra $\operatorname{dom}(S)$. The first fact is well known $[\mathrm{KuV}]$. To check the latter statement, note that for $\nu \in L_{\sharp}^{1}(\mathrm{~A}), a \in \operatorname{dom}(S)$ and $x \in \operatorname{dom}(S)$, we have

$$
\overline{\nu_{a}}(S(x))=\overline{\nu\left(a S(x)^{*}\right)}=\bar{\nu}\left(S(x) a^{*}\right)=\bar{\nu}\left(S(x) S\left(S(a)^{*}\right)\right)=\nu^{\sharp}\left(S(a)^{*} x\right) .
$$

Since $L^{1}(\mathrm{~A})$ is invariant under the right action of $\mathrm{A},\left(\nu^{\sharp}\right)_{S(a)^{*}} \in L^{1}(\mathrm{~A})$, and so it follows that $\nu_{a} \in L_{\sharp}^{1}(\mathrm{~A})$.

Let $\nu \in L_{\sharp}^{1}(\mathrm{~A})$. We need to show that

$$
E_{\mathrm{C}} R_{\nu}=R_{\nu} E_{\mathrm{C}} .
$$

As the maps above are linear and continuous, it suffices to show that

$$
E_{\mathrm{C}}\left(R_{\nu}(a)\right)=R_{\nu}\left(E_{\mathrm{C}}(a)\right), \quad a \in \mathfrak{N}_{\psi} .
$$

If $a \in \mathrm{C}$, then the formula above is an immediate consequence of right invariance of C. Let then $a \in \mathrm{C}^{\perp}$ (see the notation in Lemma 1.3). Due to Lemma 1.3 it suffices to prove that $R_{\nu}(a) \in \mathrm{C}^{\perp}$. Note first that $R_{\nu}(a) \in \mathfrak{N}_{\psi}$ because we can decompose $\nu$ into states and then use the Kadison-Schwarz inequality and right invariance of $\psi$. Put $\rho=\overline{\nu^{\sharp}}$ so that $\rho \circ S=\nu$. Let $c \in \mathrm{C} \cap \mathfrak{N}_{\psi}$ and compute:

$$
\begin{aligned}
\psi\left(c^{*} R_{\nu}(a)\right) & =\psi\left(\left(\operatorname{id}_{\mathrm{A}} \otimes \nu\right)\left(\left(c^{*} \otimes 1_{\mathrm{A}}\right) \Delta(a)\right)\right)=\nu\left(\left(\psi \otimes \operatorname{id}_{\mathrm{A}}\right)\left(\left(c^{*} \otimes 1_{\mathrm{A}}\right) \Delta(a)\right)\right) \\
& =\rho \circ S\left(\left(\psi \otimes \operatorname{id}_{\mathrm{A}}\right)\left(\left(c^{*} \otimes 1_{\mathrm{A}}\right) \Delta(a)\right)\right)=\rho\left(\left(\psi \otimes \operatorname{id}_{\mathrm{A}}\right)\left(\Delta\left(c^{*}\right)\left(a \otimes 1_{\mathrm{A}}\right)\right)\right) .
\end{aligned}
$$

The last equality holds because $\psi$ is strongly right invariant $[\mathrm{KuV}$, Proposition 5.24]. Continuing the calculation, we have

$$
\psi\left(c^{*} R_{\nu}(a)\right)=\psi\left(\left(\mathrm{id}_{\mathrm{A}} \otimes \rho\right)\left(\Delta\left(c^{*}\right)\left(a \otimes 1_{\mathrm{A}}\right)\right)\right)=\psi\left(R_{\bar{\rho}}(c)^{*} a\right)=0,
$$

as $\mathrm{C}$ is right invariant and $a \in \mathrm{C}^{\perp}$. So $R_{\nu}(a) \in \mathrm{C}^{\perp}$ as required.

A combination of Propositions 3.2 and 3.3 immediately yields the next corollary.

Corollary 3.4. If $\mathrm{C}$ is a right invariant $\psi$-expected $C^{*}$-subalgebra of $\mathrm{A}$ then it is expected (the $\psi$-preserving conditional expectation onto $\mathrm{C}$ preserves also $\phi$ ). 
The difference between the conditions in the propositions above lies of course in the fact that in the first of them we see the left Haar weight, and in the second the right Haar weight. If $A$ is unimodular, the distinction disappears and we obtain the following result. Recall that the set of right invariant expected $C^{*}$-subalgebras of $A$ is equipped with a partial order given by the set inclusion.

Theorem 3.5. Suppose that A is a unimodular coamenable locally compact quantum group. There is an order-preserving one-to-one correspondence between idempotent states $\omega$ on $\mathrm{A}$ and right invariant, expected $\mathrm{C}^{*}$-subalgebras $\mathrm{C}$ of $\mathrm{A}$. The correspondence is given by

$$
\mathrm{C}_{\omega}=L_{\omega}(\mathrm{A}), \quad \omega_{\mathrm{C}}=\epsilon \circ E_{\mathrm{C}}
$$

where $E_{\mathrm{C}}$ denotes the conditional expectation onto $\mathrm{C}$ and $\epsilon$ the counit of $\mathrm{A}$.

Proof. The existence of the correspondence is a consequence of Propositions 3.2 and 3.3. The fact that it preserves respective partial orders is easy to check.

We will show later (in Theorem 3.13) that even in the absence of unimodularity there is a one-to-one correspondence between Haar idempotents on a locally compact quantum group and right invariant expected $C^{*}$-subalgebras of a particular type. Before we do that we provide in the next theorem a criteria which characterises Haar idempotents among all idempotent states. We need another definition.

Definition 3.6. A right invariant $C^{*}$-subalgebra $\mathrm{C}$ of $\mathrm{A}$ is said to be symmetric if

$$
V^{*}(1 \otimes c) V \in M(K(H) \otimes \mathrm{C})
$$

for every $c$ in $\mathrm{C}$.

The above definition was used in $[\mathrm{Sa}]$ and a similar condition appeared already in a slightly different guise in [Tom] under the name of 'coaction symmetry': it can be interpreted as the invariance of $C$ under the natural action of the dual locally compact quantum group $\hat{\mathrm{A}}$ on $B(H)$. If $\mathrm{A}$ is the group $C^{*}$-algebra of an amenable locally compact group $G$ and $H$ is an open subgroup of $G$, then $C^{*}(H)$ is symmetric in $C^{*}(G)$ if and only if $H$ is a normal subgroup (see Section 7 in [Sa]; related facts can be also found in Theorem 3.5 of $[\mathrm{FST}]$ ).

Theorem 3.7. Let $\omega$ be an idempotent state on $\mathrm{A}$ and let $\mathrm{C}_{\omega}=L_{\omega}(\mathrm{A})$. Then the following are equivalent:

(i) $\omega$ is a Haar idempotent;

(ii) $\mathrm{C}_{\omega}$ is symmetric;

(iii) $N_{\omega}:=\left\{a \in \mathrm{A}: \omega\left(a^{*} a\right)=0\right\}$ is an ideal (equivalently $a^{*}$-subspace).

Proof. (i) $\Longrightarrow$ (ii) Suppose that B is a compact quantum subgroup of $A$, that $\pi_{B}$ is a corresponding morphism and that $\omega=h_{\mathrm{B}} \circ \pi_{\mathrm{B}}$, where $h_{\mathrm{B}}$ is the Haar state of $\mathrm{B}$. Then Theorem 10 of $[\mathrm{Sa}]$ states that $\mathrm{C}_{\omega}=L_{\omega}(\mathrm{A})$ is symmetric.

(ii) $\Longrightarrow$ (i) The left invariant $C^{*}$-subalgebra $C_{\omega}$ gives rise to a compact quantum subgroup $B$ of $A$ by Theorem 2 of $[\mathrm{Sa}]$. Let $\pi_{B}: A \rightarrow B$ be the associated morphism and let $h_{\mathrm{B}}$ be the Haar state of B. Now the Haar idempotent $\gamma:=h_{\mathrm{B}} \circ \pi_{\mathrm{B}}$ gives rise to another right invariant $C^{*}$-subalgebra $\mathrm{C}_{\gamma}=L_{\gamma}(\mathrm{A})$. But since $\mathrm{C}_{\omega}$ admits a conditional expectation $L_{\omega}$ satisfying the invariance condition $\left(L_{\omega} \otimes \mathrm{id}_{\mathrm{A}}\right) \Delta=\Delta L_{\omega}$, Theorem 11 of $[\mathrm{Sa}]$ implies that $\mathrm{C}_{\omega}=\mathrm{C}_{\gamma}$. Now both $L_{\omega}$ and $L_{\gamma}$ are $\phi$-preserving conditional expectations onto the same $C^{*}$-subalgebra, so by the uniqueness of 
conditional expectations preserving a fixed faithful weight, $L_{\omega}=L_{\gamma}$. The last equality yields $\gamma=\omega$, so that $\omega$ is a Haar idempotent.

(i) $\Longrightarrow$ (iii) Let $\omega$ be a Haar idempotent, so that there exists a compact quantum subgroup B of A such that $\omega=h_{\mathrm{B}} \circ \pi_{\mathrm{B}}$. Since the Haar state $h_{\mathrm{B}}$ is faithful, we have

$$
\begin{aligned}
a \in N_{\omega} & \Longleftrightarrow \omega\left(a^{*} a\right)=0 \Longleftrightarrow h_{\mathrm{B}}\left(\pi_{\mathrm{B}}(a)^{*} \pi_{\mathrm{B}}(a)\right)=0 \Longleftrightarrow \pi_{\mathrm{B}}(a)=0 \\
& \Longleftrightarrow \pi_{\mathrm{B}}\left(a^{*}\right)=0 \Longleftrightarrow \omega\left(a a^{*}\right)=0 \Longleftrightarrow a^{*} \in N_{\omega} .
\end{aligned}
$$

(iii) $\Longrightarrow$ (i) Suppose that $N_{\omega}$ is an ideal. Let B denote the $C^{*}$-algebra $\mathrm{A} / N_{\omega}$, and let $\pi_{\omega}: \mathrm{A} \rightarrow \mathrm{B}$ be the quotient map. We claim that $\mathrm{B}$ is unital. Let $e \in \mathrm{A}_{+}$ such that $\omega(e)=1$. To show that $\pi_{\omega}\left(L_{\omega}(e)\right)$ is a unit for $\mathrm{B}$, we need to show that $a-a L_{\omega}(e)$ and $a-L_{\omega}(e) a$ are in $N_{\omega}$ for every $a$ in A. Since $L_{\omega}(e)$ is in the multiplicative domain of $\omega$ by Lemma 2.5,

$$
\begin{aligned}
\omega\left(\left(a-a L_{\omega}(e)\right)^{*}\left(a-a L_{\omega}(e)\right)\right) & \\
= & \omega\left(a^{*} a-a^{*} a L_{\omega}(e)-L_{\omega}\left(e^{*}\right) a^{*} a+L_{\omega}\left(e^{*}\right) a^{*} a L_{\omega}(e)\right) \\
= & \omega\left(a^{*} a\right)\left(1-\omega\left(L_{\omega}(e)\right)-\omega\left(L_{\omega}(e)\right)+\omega\left(L_{\omega}(e)\right)^{2}\right) \\
= & \omega\left(a^{*} a\right)\left(1-2 \omega(e)+\omega(e)^{2}\right)=0 .
\end{aligned}
$$

Since $N_{\omega}$ is self-adjoint, also $a-L_{\omega}(e) a=\left(a^{*}-a^{*} L_{\omega}(e)\right)^{*}$ is in $N_{\omega}$.

The proof continues from now on as in the compact case [FST], with some necessary modifications. Recall that a positive map between $C^{*}$-algebras is said to be faithful if it maps positive non-zero elements into non-zero elements. Let $\left(e_{i}\right)_{i \in I}$ denote an approximate unit in A. A standard use of the Cauchy-Schwarz inequality implies that if $a \in N_{\omega}$, then $\omega\left(e_{i} a\right)=0$ for all $i \in I$, and so $\omega(a)=0$. Let $\mu \in \mathrm{B}^{*}$ be a linear functional such that $\mu \circ \pi_{\omega}=\omega$. It is obviously positive and

$$
\mu\left(1_{\mathrm{B}}\right)=\mu\left(\pi_{\omega}\left(L_{\omega}(e)\right)\right)=\omega(e)=1,
$$

so $\mu$ is a state.

Since $\mu \circ \pi_{\omega}=\omega$ and $\operatorname{ker} \pi_{\omega}=N_{\omega}$ it follows that $\mu$ is faithful. Let $x$ in $\mathrm{B} \otimes \mathrm{B}$ be positive. If $\left(\operatorname{id}_{\mathrm{B}} \otimes \mu\right)(x)=0$, then $\mu\left(\left(\nu \otimes \operatorname{id}_{\mathrm{B}}\right)(x)\right)=0$ for any state $\nu \in \mathrm{B}^{*}$, and so $\left(\nu \otimes \operatorname{id}_{\mathrm{B}}\right)(x)=0$. This in turn implies that $x=0$ (replace $\nu$ by an arbitrary continuous functional, apply another one of these and use the fact that tensor products of functionals in $\mathrm{B}^{*}$ separate points in $\mathrm{B} \otimes \mathrm{B}$ ). Thus we have shown that $\mathrm{id}_{\mathrm{B}} \otimes \mu: \mathrm{B} \otimes \mathrm{B} \rightarrow \mathrm{B}$ is faithful. The faithfulness of $\mu \otimes \mu \in(\mathrm{B} \otimes \mathrm{B})^{*}$ is now an easy consequence.

Suppose now that $a \in N_{\omega}$. We have then

$$
0=\omega\left(a^{*} a\right)=(\omega \otimes \omega) \circ \Delta\left(a^{*} a\right)=(\mu \otimes \mu) \circ\left(\pi_{\omega} \otimes \pi_{\omega}\right)\left(\Delta\left(a^{*} a\right)\right),
$$

so also $\left(\pi_{\omega} \otimes \pi_{\omega}\right)\left(\Delta\left(a^{*} a\right)\right)=0$ (note that we use here a strict extension of $\pi_{\omega} \otimes \pi_{\omega}$, which also takes values in $\mathrm{B} \otimes \mathrm{B})$. The last statement implies that $\left(\pi_{\omega} \otimes \pi_{\omega}\right) \Delta(a)=0$.

The computation in the last paragraph implies that we can define a map $\Delta_{\mathrm{B}}$ : $\mathrm{B} \rightarrow \mathrm{B} \otimes \mathrm{B}$ via the formula

$$
\Delta_{\mathrm{B}} \circ \pi_{\omega}=\left(\pi_{\omega} \otimes \pi_{\omega}\right) \circ \Delta .
$$

The fact that $\Delta_{\mathrm{B}}$ is a nondegenerate (hence unital) ${ }^{*}$-homomorphism follows immediately from the analogous properties of $\Delta$. Once we know that $\Delta_{B}$ is nondegenerate we can check that it is coassociative (all maps involved in the calculations are strict 
so there are no problems in passing to multiplier algebras). Finally the quantum cancellation properties of $B$ follow from obvious equalities of the type

$$
\left(\mathrm{B} \otimes 1_{\mathrm{B}}\right) \Delta_{\mathrm{B}}(\mathrm{B})=\left(\pi_{\omega} \otimes \pi_{\omega}\right)\left(\left(\mathrm{A} \otimes 1_{\mathrm{A}}\right) \Delta(\mathrm{A})\right)
$$

and the fact that the quantum cancellation properties hold for $A$. Thus $\left(B, \Delta_{B}\right)$ is a compact quantum group. It is easy to check that $\mu$ defined above is an idempotent state on $B$. As it is faithful, it coincides with the Haar state of $B$ by $\left[\mathrm{Wo}_{3}\right]$.

The following corollary is an immediate consequence of the equivalence of (i) and (iii). In the commutative case it gives the Kawada-Itô Theorem.

Corollary 3.8. If an idempotent state on a coamenable locally compact quantum group A is tracial, then it is a Haar idempotent. In particular if A is commutative, then all idempotent states on $\mathrm{A}$ are Haar idempotents.

The equivalence of conditions (i) $\Longleftrightarrow($ ii) is essentially contained in [Sa]; in the case of von Neumann algebraic compact quantum groups it was shown in [Tom].

Below we identify the ideal $N_{\omega}$ appearing in Theorem 3.7 with an ideal constructed in $[\mathrm{Sa}]$, but we need some terminology first. Let $\mathrm{C}$ be a right invariant $\mathrm{C}^{*}$-subalgebra of A. We say that a nondegenerate representation $\rho$ of $\mathrm{A}$ on a Hilbert space $H_{\rho}$ is C-trivial if

$$
\rho(c)=\epsilon(c) 1_{H_{\rho}} \quad \text { for every } c \text { in } \mathbf{C} .
$$

Define $J_{\mathrm{C}}=\bigcap_{\rho} \operatorname{ker} \rho$ where the intersection is taken over the equivalence classes of nondegenerate $\mathrm{C}$-trivial representations of $\mathrm{A}$.

Proposition 3.9. Let $\omega$ be a Haar idempotent and let $\mathrm{B}$ be a compact quantum subgroup of $\mathrm{A}$ such that $\omega=h_{\mathrm{B}} \circ \pi_{\mathrm{B}}$. Then $N_{\omega}=J_{\mathrm{C}_{\omega}}$ where $\mathrm{C}_{\omega}=L_{\omega}(\mathrm{A})$.

Proof. By the construction in Section 5 of [Sa], the right invariant $C^{*}$-subalgebra associated with the compact quantum subgroup $B$ is precisely $C_{\omega}$. Then the ideal $J_{\mathrm{C}_{\omega}}$, again by construction, is the kernel of the map $\pi_{\mathrm{B}^{\prime}}: \mathrm{A} \rightarrow \mathrm{B}^{\prime}$, where $\mathrm{B}^{\prime}$ is the compact quantum subgroup associated with $\mathrm{C}_{\omega}[\mathrm{Sa}$, Theorem 2]. Now the uniqueness result [Sa, Theorem 12] says that $B$ is isomorphic to $B^{\prime}$. In fact, as is shown in the proof of [Sa, Theorem 12], $\operatorname{ker} \pi_{\mathrm{B}}=\operatorname{ker} \pi_{\mathrm{B}^{\prime}}$. But $J_{\mathrm{C}_{\omega}}=\operatorname{ker} \pi_{\mathrm{B}^{\prime}}$ and $N_{\omega}=\operatorname{ker} \pi_{\mathrm{B}^{\prime}}$ by (3.4), so it follows that $N_{\omega}=J_{\mathrm{C}_{\omega}}$.

The remainder of this section will be devoted to the extension of Theorem 3.5 to non-unimodular locally compact quantum groups in the case of Haar idempotents. The proof of Theorem 3.5 suggests that the only missing ingredient needed for such an extension is the fact that the conditional expectation $L_{\omega}$ preserves not only the left Haar weight $\phi$, but also the right Haar weight $\psi$. It is natural to expect that to show this we need to exploit the modular element facilitating the passage between $\phi$ and $\psi$.

The proof will be split into a series of lemmas. The first one is probably well known and does not involve any notions related to quantum groups.

Lemma 3.10. Let $\mathrm{D}$ be a $C^{*}$-algebra, let $\rho \in \mathrm{D}^{*}$ be a state and let $\left(\pi, H_{\rho}, \Omega\right)$ be the GNS construction for $\rho$. Denote by $\rho^{\prime} \in(\pi(\mathrm{D}))^{*}$ the vector state associated with $\Omega$ (so that $\rho=\rho^{\prime} \circ \pi$ ). Then $\rho^{\prime}$ is faithful if and only if the null space $N_{\rho}:=\{d \in \mathrm{D}$ : $\left.\rho\left(d^{*} d\right)=0\right\}$ is an ideal. 
Proof. Let $d \in \mathrm{D}$. On one hand we have the following string of equivalences:

$$
\begin{aligned}
\pi(d)=0 & \Longleftrightarrow \forall_{c \in \mathrm{D}} \pi(d) \pi(c) \Omega=0 \Longleftrightarrow \forall_{c \in \mathrm{D}}\left\langle\Omega, \pi(c)^{*} \pi(d)^{*} \pi(d) \pi(c) \Omega\right\rangle=0 \\
& \Longleftrightarrow \forall_{c \in \mathrm{D}} \rho\left(c^{*} d^{*} d c\right)=0 \Longleftrightarrow \forall_{c \in \mathrm{D}} d c \in N_{\rho} .
\end{aligned}
$$

On the other hand $\rho^{\prime}\left(\pi(d)^{*} \pi(d)\right)=\rho\left(d^{*} d\right)=0$ if and only if $d \in N_{\rho}$.

The next lemma will be used to show that Haar idempotents are invariant under the suitably understood action of the modular element. Classically this corresponds to the fact that the modular function of a locally compact group $G$ is the constant function 1 when restricted to any compact subgroup of $G$ (because it is a continuous homomorphism into the non-negative reals).

Lemma 3.11. Let $\omega \in \mathrm{A}^{*}$ be a Haar idempotent and let $\left(\pi, H_{\omega}, \Omega\right)$ be the GNS construction for $\omega$. Then the $C^{*}$-algebra $\pi(\mathrm{A})$ is unital. If $t$ is a positive selfadjoint operator affiliated with $\mathrm{A}$ such that $\Delta(t)=t \otimes t$, then the positive functional $\omega_{t} \in \mathrm{A}^{*}$ defined by the formula

$$
\omega_{t}(a)=\langle\Omega, \pi(t) \pi(a) \pi(t) \Omega\rangle, \quad a \in \mathrm{A},
$$

is either zero or an idempotent state. Moreover, if $t$ is strictly positive, then actually $\pi(t)=1$ and $\omega_{t}=\omega$.

Proof. Similarly to the previous lemma let $\omega^{\prime} \in \pi(\mathrm{A})^{*}$ be such that $\omega=\omega^{\prime} \circ \pi$. Choose $e \in \mathrm{A}_{+}$such that $\omega(e)=1$. We claim that $\pi\left(L_{\omega}(e)\right)$ is a unit for $\pi(\mathrm{A})$. Indeed, by the implication (i) $\Longrightarrow$ (iii) of Theorem 3.7 and the previous lemma it suffices to show that both $\pi\left(L_{\omega}(e) a\right)-\pi(a)$ and $\pi\left(a L_{\omega}(e)\right)-\pi(a)$ belong to $\{b \in$ $\left.\pi(\mathrm{A}): \omega^{\prime}\left(b^{*} b\right)=0\right\}$ for arbitrary $a \in \mathrm{A}$. This however can be established exactly as in the proof of the implication (iii) $\Longrightarrow$ (i) in Theorem 3.7.

Since $\pi$ is a nondegenerate ${ }^{*}$-homomorphism and $t$ is affiliated with $\mathrm{A}$, it follows that $\pi(t)$ is affiliated with $\pi(\mathrm{A})$. But $\pi(\mathrm{A})$ is unital, so $\pi(t) \in \mathrm{A}$. In particular $\pi(t)$ is bounded, and so the formula (3.6) defines a bounded functional on A.

Let $D_{t}=\{a \in \mathrm{A}$ : at is bounded $\}$. Let us note that since $t$ is positive, $D_{t}=$ $(\operatorname{dom}(t))^{*}$. Indeed, suppose first that at is bounded. For every $b$ in $\operatorname{dom}(t)$ we have $a(t b)=(a t) b$. Therefore, by definition, $a^{*} \in \operatorname{dom}\left(t^{*}\right)$ and $t^{*} a^{*}=(a t)^{*}$. Since $t=t^{*}$, we have $t a^{*}=(a t)^{*}$. Conversely, suppose that $a^{*} \in \operatorname{dom}(t)$. Then for every $b$ in $\operatorname{dom}(t)$ we have $a(t b)=\left(t a^{*}\right)^{*} b$, so $a t=\left(t a^{*}\right)^{*}$ on $\operatorname{dom}(t)$. Hence $a t$ is bounded.

For $a \in D_{t}$ we have

$$
\begin{aligned}
\left(\omega_{t} \star \omega_{t}\right)\left(a^{*} a\right) & =\left\langle\Omega \otimes \Omega,(\pi \otimes \pi)(t \otimes t)(\pi \otimes \pi)\left(\Delta\left(a^{*} a\right)\right)(\pi \otimes \pi)(t \otimes t)(\Omega \otimes \Omega)\right\rangle \\
& =\left\langle\Omega \otimes \Omega,(\pi \otimes \pi)(\Delta(t))(\pi \otimes \pi)\left(\Delta\left(a^{*} a\right)\right)(\pi \otimes \pi)(\Delta(t))(\Omega \otimes \Omega)\right\rangle .
\end{aligned}
$$

By (1.1),

$$
(\pi \otimes \pi)(\Delta(t))\left((\pi \otimes \pi)\left(\Delta\left(a^{*}\right)\right) u\right)=(\pi \otimes \pi)\left(\Delta\left(t a^{*}\right)\right) u
$$

for every $u \in \pi(\mathrm{A}) \otimes \pi(\mathrm{A})$. On both sides of the equation we have bounded operators evaluated at $u$ so

$$
(\pi \otimes \pi)(\Delta(t))(\pi \otimes \pi)\left(\Delta\left(a^{*}\right)\right)=(\pi \otimes \pi)\left(\Delta\left(t a^{*}\right)\right)
$$

in $\pi(\mathrm{A}) \otimes \pi(\mathrm{A})$. It also follows that

$$
(\pi \otimes \pi)(\Delta(a))(\pi \otimes \pi)(\Delta(t))=(\pi \otimes \pi)(\Delta(a t)) .
$$


Inserting these into the expansion of $\left(\omega_{t} \star \omega_{t}\right)\left(a^{*} a\right)$, we have

$$
\begin{aligned}
\left(\omega_{t} \star \omega_{t}\right)\left(a^{*} a\right) & =\left\langle\Omega \otimes \Omega,(\pi \otimes \pi)\left(\Delta\left(t a^{*} a t\right)\right)(\Omega \otimes \Omega)\right\rangle=(\omega \otimes \omega)\left(\Delta\left(t a^{*} a t\right)\right) \\
& =\omega\left(t a^{*} a t\right)=\left\langle\Omega, \pi\left(t a^{*} a t\right) \Omega\right\rangle=\omega_{t}\left(a^{*} a\right)
\end{aligned}
$$

As $\left\{a^{*} a: a \in D_{t}\right\}$ is dense in $\mathrm{A}_{+}$, by linearity and continuity we deduce that $\omega_{t} \star \omega_{t}=\omega_{t}$. Hence $\omega_{t}$ is either 0 or a state.

Suppose now that $t$ is strictly positive. Then $t^{-1}$ is a strictly positive operator affiliated with $\mathrm{A}$. Hence $\pi\left(t^{-1}\right) \in \pi(\mathrm{A})$ so it follows that $\pi(t)$ is invertible with an inverse in $\pi(\mathrm{A})$. Let then $a \in \mathrm{A}$ be such that $\pi(a)=\pi\left(t^{-1}\right)$. Then $\omega_{t}\left(a^{2}\right)=\omega^{\prime}(1)=$ 1. So $\omega_{t}$ is nonzero and hence a state. Therefore $\omega^{\prime}\left(\pi(t)^{2}\right)=\omega_{t}(1)=1$.

By functional calculus, $t^{2}$ is a strictly positive element affiliated with A. Moreover

$$
\Delta\left(t^{2}\right)=\Delta(t)^{2}=(t \otimes t)^{2}=t^{2} \otimes t^{2}
$$

by (1.2) and $\left[\mathrm{Ku}_{2}\right.$, Proposition 13.16]. It follows that the preceding argument can be applied to $t^{2}$ instead of $t$, and so $\omega^{\prime}\left(\pi(t)^{4}\right)=1$. Hence

$$
\omega^{\prime}\left(\left(\pi(t)^{2}-1\right)^{*}\left(\pi(t)^{2}-1\right)\right)=0,
$$

and by the faithfulness of $\omega^{\prime}$ (Lemma 3.10) it follows that $\pi(t)^{2}=1$. Positivity and uniqueness of square roots imply that $\pi(t)=1$ and $\omega_{t}=\omega$.

Proposition 3.12. Let $\omega \in \mathrm{A}^{*}$ be a Haar idempotent. The conditional expectation $L_{\omega}$ preserves the right Haar weight.

Proof. Denote the modular element affiliated with A by $\delta$. To simplify the notation we write $x:=\delta^{\frac{1}{2}}$. So $x$ is defined by functional calculus of affiliated elements and is itself affiliated with $A$. We need to show that the completely positive operator $L_{\omega}: \mathrm{A} \rightarrow \mathrm{A}$ preserves the right invariant weight $\psi$. Our approach is based on the fact that formally

$$
\psi=\phi(x \cdot x)
$$

Following Kustermans $\left[\mathrm{Ku}_{1}\right.$, Section 8] and Vaes $\left[\mathrm{Va}_{1}\right.$, p. 325], put

$$
D_{x}^{\phi}=\left\{a \in \mathrm{A}: a x \text { is bounded and } \overline{a x} \in \mathfrak{N}_{\phi}\right\} .
$$

(The overline denotes closure.) Then $D_{x}^{\phi}$ is a dense left ideal in A [ $\mathrm{Ku}_{1}$, Result 8.6]. Let $a \in D_{x}^{\phi}$. It follows from [ $\mathrm{Ku}_{1}$, Corollary 8.35] that $a \in \mathfrak{N}_{\psi}$ (because $\psi=\phi(x \cdot x)$ ). Moreover, $x a^{*}=(a x)^{*} \in \mathfrak{N}_{\phi}^{*}$ (in particular, $x a^{*}$ is defined everywhere and is in A).

We shall show that any $a$ in $D_{x}^{\phi}$ satisfies

$$
\overline{x L_{\omega}\left(a^{*} a\right) x}=L_{\omega}(\overline{a x} * \overline{a x})
$$

In particular, $L_{\omega}\left(a^{*} a\right) \in \mathfrak{M}_{\psi}^{+}$because $\overline{a x} \overline{a x}_{\overline{a x}} \in \mathfrak{M}_{\phi}$ (by $\left[\mathrm{Ku}_{1}\right.$, Corollary 8.35] again). Then we may calculate

$$
\psi\left(a^{*} a\right)=\phi\left(\overline{x a^{*} a x}\right)=\phi\left(\overline{a x}^{*} \overline{a x}\right)=\phi\left(L_{\omega}\left(\overline{a x}^{*} \overline{a x}\right)\right)=\phi\left(\overline{x L_{\omega}\left(a^{*} a\right) x}\right)=\psi\left(L_{\omega}\left(a^{*} a\right)\right) .
$$

Note that the elements of the form $a^{*} a, a \in D_{x}^{\phi}$, are dense in $\mathrm{A}_{+}$, and so it follows that $\gamma:=\psi \circ L_{\omega}$ is a nonzero, densely defined, lower semicontinuous weight on A. We show next that $\gamma$ is right invariant. For any $a \in \mathfrak{M}_{\gamma}$ and a state $\eta \in A_{+}^{*}$, we have

$$
\gamma\left(\left(\operatorname{id}_{\mathrm{A}} \otimes \eta\right)(\Delta(a))\right)=\psi\left(L_{\omega} R_{\eta}(a)\right)=\psi\left(R_{\eta} L_{\omega}(a)\right)
$$

Now we use the fact that for invariant weights the invariance holds in a strong sense: $\psi\left(R_{\eta}(x)\right)=\psi(x)$ for all $x \in \mathrm{A}_{+}$. So we continue:

$$
\gamma\left(\left(\operatorname{id}_{\mathrm{A}} \otimes \eta\right)(\Delta(a))\right)=\psi\left(L_{\omega}(a)\right)=\gamma(a) .
$$


By the uniqueness of right Haar weight [KuV, Theorem 7.15], $\gamma$ is a scalar multiple of $\psi$. The scalar has to be 1 because $\gamma$ and $\psi$ coincide on a dense subset. Therefore $L_{\omega}$ preserves the right Haar weight.

We proceed to prove the identity (3.7). Since the modular element $\delta$ is strictly positive and $\Delta(\delta)=\delta \otimes \delta[\mathrm{KuV}$, Proposition 7.12], it follows from functional calculus and $\left[\mathrm{Ku}_{2}\right.$, Proposition 13.16$]$ that

$$
\Delta\left(\delta^{z}\right)=\delta^{z} \otimes \delta^{z}
$$

for any $z$ in $\mathbb{C}$. Let $\left(\pi, H_{\omega}, \Omega\right)$ be the GNS construction for $\omega$ and let $\omega^{\prime} \in \pi(\mathrm{A})^{*}$ the vector state associated with $\Omega$. Then Lemma 3.11 implies that

$$
\omega(a)=\omega^{\prime}\left(\pi(x)^{-1} \pi(a) \pi(x)^{-1}\right) \quad(a \in \mathrm{A}),
$$

To make the presentation more transparent, we denote below the functional defined by the right-hand side of the identity above as $\widetilde{\omega}$.

Let $a \in D_{x}^{\phi}$. As noted, $x a^{*} a x$ is bounded and densely defined: its extension is $\left(x a^{*}\right) \overline{a x}=\left(x a^{*}\right)\left(x a^{*}\right)^{*}$. Now

$$
\begin{aligned}
& L_{\widetilde{\omega}}\left(\overline{x a^{*} a x}\right)=L_{\widetilde{\omega}}\left(x a^{*} \overline{a x}\right) \\
& \quad=\left(\omega^{\prime} \otimes \mathrm{id}_{\mathrm{A}}\right)\left(\left(\pi(x)^{-1} \otimes 1_{\mathrm{A}}\right)\left(\pi \otimes \mathrm{id}_{\mathrm{A}}\right)\left(\Delta\left(x a^{*} \overline{a x}\right)\right)\left(\pi(x)^{-1} \otimes 1_{\mathrm{A}}\right)\right) \\
& \quad=\left(\omega^{\prime} \otimes \mathrm{id}_{\mathrm{A}}\right)\left(\left(\pi(x)^{-1} \otimes 1_{\mathrm{A}}\right)\left(\pi \otimes \mathrm{id}_{\mathrm{A}}\right)\left(\Delta\left(x a^{*}\right)\right)\left(\pi \otimes \mathrm{id}_{\mathrm{A}}\right)(\Delta(\overline{a x}))\left(\pi(x)^{-1} \otimes 1_{\mathrm{A}}\right)\right)
\end{aligned}
$$

Now both $\Delta$ and $\pi \otimes \operatorname{id}_{\mathrm{A}}$ are nondegenerate ${ }^{*}$-homomorphisms so $\left(\pi \otimes \operatorname{id}_{\mathrm{A}}\right) \Delta(x)$ is affiliated with $\pi(\mathrm{A}) \otimes \mathrm{A}$ and

$$
\left(\pi \otimes \operatorname{id}_{\mathrm{A}}\right)\left(\Delta\left(x a^{*}\right)\right) u=\left(\pi \otimes \operatorname{id}_{\mathrm{A}}\right)(\Delta(x))\left(\left(\pi \otimes \operatorname{id}_{\mathrm{A}}\right) \Delta\left(a^{*}\right) u\right)
$$

for every $u$ in $\pi(\mathrm{A}) \otimes \mathrm{A}$ (see (1.1)). Since the linear span of $(\pi(\mathrm{A}) \otimes \mathrm{A})\left(H_{\omega} \otimes H\right)$ is dense in $H_{\omega} \otimes H$, it follows that

$$
\left(\pi \otimes \operatorname{id}_{\mathrm{A}}\right)\left(\Delta\left(x a^{*}\right)\right)=\left(\pi \otimes \operatorname{id}_{\mathrm{A}}\right)(\Delta(x))\left(\pi \otimes \operatorname{id}_{\mathrm{A}}\right)\left(\Delta\left(a^{*}\right)\right)
$$

in $B\left(H_{\omega} \otimes H\right)$.

Moreover,

$$
\left(\pi \otimes \operatorname{id}_{\mathrm{A}}\right)(\Delta(x))=\pi(x) \otimes x
$$

because $\Delta(x)=x \otimes x$ (apply Theorem 6.1 of [NaW] and Theorem 1.2 of [ $\left[\mathrm{Wo}_{1}\right]$ ). So we have

$$
\left(\pi \otimes \operatorname{id}_{\mathrm{A}}\right)\left(\Delta\left(x a^{*}\right)\right)=(\pi(x) \otimes x)\left(\left(\pi \otimes \operatorname{id}_{\mathrm{A}}\right) \Delta\left(a^{*}\right)\right) .
$$

Finally,

$$
\left(\pi(x)^{-1} \otimes 1_{\mathrm{A}}\right)\left(\pi \otimes \operatorname{id}_{\mathrm{A}}\right)\left(\Delta\left(x a^{*}\right)\right)=\left(1_{\mathrm{A}} \otimes x\right)\left(\left(\pi \otimes \operatorname{id}_{\mathrm{A}}\right) \Delta\left(a^{*}\right)\right) .
$$

On the other hand, since $\overline{a x}=\left(x a^{*}\right)^{*}$,

$$
\left(\pi \otimes \operatorname{id}_{\mathrm{A}}\right)(\Delta(\overline{a x}))\left(\pi(x)^{-1} \otimes 1_{\mathrm{A}}\right)=\left(\left(\pi(x)^{-1} \otimes 1_{\mathrm{A}}\right)\left(\pi \otimes \operatorname{id}_{\mathrm{A}}\right)\left(\Delta\left(x a^{*}\right)\right)\right)^{*},
$$

which is, by (3.9), the closure of the bounded densely defined operator

$$
\left(\pi \otimes \operatorname{id}_{\mathrm{A}}\right)(\Delta(a))\left(1_{\mathrm{A}} \otimes x\right) .
$$


Continuing from (3.8), we have

$$
\begin{aligned}
& L_{\widetilde{\omega}}\left(\overline{x a^{*} a x}\right)=\left(\omega^{\prime} \otimes \operatorname{id}_{\mathrm{A}}\right)\left(\left(1_{\mathrm{A}} \otimes x\right)\left(\left(\pi \otimes \operatorname{id}_{\mathrm{A}}\right)\left(\Delta\left(a^{*}\right)\right) \overline{\left(\pi \otimes \operatorname{id}_{\mathrm{A}}\right)(\Delta(a))\left(1_{\mathrm{A}} \otimes x\right)}\right)\right. \\
& =\overline{x\left(\omega^{\prime} \otimes \operatorname{id}_{\mathrm{A}}\right)\left(\left(\pi \otimes \mathrm{id}_{\mathrm{A}}\right)\left(\Delta\left(a^{*}\right)\right)\left(\pi \otimes \operatorname{id}_{\mathrm{A}}\right)(\Delta(a))\right) x} \\
& =\overline{x\left(\omega^{\prime} \otimes \operatorname{id}_{\mathrm{A}}\right)\left(\left(\pi \otimes \operatorname{id}_{\mathrm{A}}\right)\left(\Delta\left(a^{*} a\right)\right)\right) x} \\
& =\overline{x L_{\omega}\left(a^{*} a\right) x} \text {. }
\end{aligned}
$$

Therefore (3.7) holds.

Theorem 3.13. Let $\mathrm{A}$ be a coamenable locally compact quantum group. There is an order-preserving one-to-one correspondence between Haar idempotents $\omega$ on $\mathrm{A}$ and symmetric, right invariant, expected $C^{*}$-subalgebras $\mathrm{C}$ of $\mathrm{A}$. The correspondence is given by

$$
\mathrm{C}_{\omega}=L_{\omega}(\mathrm{A}), \quad \omega_{\mathrm{C}}=\epsilon \circ E_{\mathrm{C}},
$$

where $E_{\mathrm{C}}$ denotes the conditional expectation onto $\mathrm{C}$ and $\epsilon$ the counit of $\mathrm{A}$.

Proof. The theorem is a consequence of Propositions 3.2, 3.3, 3.12 and Theorem 3.7.

Let $\mathrm{A}$ be as in the previous theorem. It is shown in [Sa] that there is a one-toone correspondence between compact quantum subgroups of $A$ and symmetric, right invariant $C^{*}$-subalgebras $\mathrm{C}$ of $\mathrm{A}$ that have a conditional expectation $E_{\mathrm{C}}: \mathrm{A} \rightarrow \mathrm{C}$ such that $\left(E_{\mathrm{C}} \otimes \mathrm{id}_{\mathrm{A}}\right) \circ \Delta=\Delta \circ E_{\mathrm{C}}$. (We have changed 'left invariant' of [Sa] to 'right invariant' to conform with the current terminology.) The previous theorem replaces the condition $\left(E_{\mathrm{C}} \otimes \mathrm{id}_{\mathrm{A}}\right) \circ \Delta=\Delta \circ E_{\mathrm{C}}$ with a formally weaker one: that $E_{\mathrm{C}}$ is invariant under both left and right Haar weights. The latter condition is also much more natural.

We would like to finish the section with a few more general remarks. Given a locally compact quantum group $\mathrm{A}$, the determination of all of its compact quantum subgroups is a difficult technical problem; in fact the answer to this question does not seem to be known for any of the fundamental examples of genuine quantum groups such as the quantum $E(2)\left[\mathrm{Wo}_{2}\right]$, quantum $a z+b\left[\mathrm{Wo}_{4}\right]$ or quantum $\widehat{S U(1,1)}[\mathrm{GKK}]$. Our results suggest a possible approach to this question via first determining all idempotent states on these quantum groups. This idea was successfully applied in [FST] in the context of compact quantum groups, for example for $U_{q}(2)$. Of course to apply the techniques developed in this paper, we need to know that the quantum group in question is coamenable. The result below is probably very well-known to the experts, but as we could not find an explicit reference, we sketch a short proof.

Theorem 3.14. The locally compact quantum groups quantum E(2), quantum $a z+b$ and quantum $a x+b$ are coamenable.

Proof. As all the quantum groups in question were originally defined using the language of multiplicative unitaries, we first need to note that they all fit into the setup of $[\mathrm{KuV}]$, i.e. that they admit faithful invariant KMS-weights. For the last two examples this has been established in $\left[\mathrm{Wo}_{5}\right]$ and in $[\mathrm{VD}]$; for the first in $\left[\mathrm{Ba}_{2}\right]$ and in $[\mathrm{VD}]$.

Hence it suffices to observe that all these locally compact quantum groups admit bounded counits. In each case the potential definition for the counit can be guessed 
from the explicit formulas for the coproduct in terms of the (unbounded) generators. So for example for quantum $a z+b$ we see that the counit, if it exists, must take value 1 at $a$ and 0 at $b$ (precisely speaking we should consider here an extension of the potential counit to the algebra of affiliated elements, but this does not affect the argument). The fact that in each of the three cases the deduced prescription indeed defines a continuous character follows from the universal properties of the underlying $C^{*}$-algebras with respect to specific commutation relations (these universal properties are established respectively in Theorem 1.1 of $\left[\mathrm{Wo}_{2}\right]$, Proposition 4.2 of $\left[\mathrm{Wo}_{4}\right]$ and Proposition 3.2 of [WoZ]). We leave the details to the reader.

Note that the coamenability of quantum $E(2)$ has been established in the $\mathrm{PhD}$ thesis of Jacobs [Jac] (the terminology used there is different), and its counit is also explicitly mentioned in $\left[\mathrm{Wo}_{2}\right]$. We do not know whether the quantum $\widetilde{S U(1,1)}$ is coamenable.

\section{Further Properties of Right INVARIANt $C^{*}$-SUbalgebras}

In this short section we show that the algebras of the form $L_{\omega}(\mathrm{A})$, where $\omega$ is an idempotent state on a locally compact quantum group $A$, admit a natural coaction of A. Such subalgebras generalise quantum homogenous spaces which are naturally associated with compact quantum subgroups of A (see Proposition 4.1 or Section 5 in $[\mathrm{Sot}])$.

Assume again that $A$ is a coamenable locally compact quantum group and let $\omega \in A^{*}$ be a Haar idempotent corresponding to a fixed compact quantum subgroup of $\mathrm{A}$, denoted by $\mathrm{B}$. The algebra $\mathrm{C}_{\omega}=L_{\omega}(\mathrm{A})$ should be thought of as the algebra of functions in $A$ which are invariant under the action of $B$. This is formalised in the following proposition.

Proposition 4.1. Suppose that $\mathrm{B}$ is a compact quantum subgroup of $\mathrm{A}$ and $\pi$ is the surjective morphism from $\mathrm{A}$ to $\mathrm{B}$. Let $\omega=h_{\mathrm{B}} \circ \pi$ be the Haar idempotent associated with B. Then

$$
L_{\omega}(\mathrm{A})=\left\{a \in \mathrm{A}:\left(\pi \otimes \operatorname{id}_{\mathrm{A}}\right)(\Delta(a))=1_{\mathrm{B}} \otimes a\right\} .
$$

Proof. Fix $a \in$ A. If $\left(\pi \otimes \operatorname{id}_{\mathrm{A}}\right)(\Delta(a))=1_{\mathrm{B}} \otimes a$, then

$$
L_{\omega}(a)=\left(h_{\mathrm{B}} \otimes \operatorname{id}_{\mathrm{A}}\right)\left(\left(\pi \otimes \operatorname{id}_{\mathrm{A}}\right)(\Delta(a))\right)=\left(h_{\mathrm{B}} \otimes \operatorname{id}_{\mathrm{A}}\right)\left(1_{\mathrm{B}} \otimes a\right)=a,
$$

so $a \in L_{\omega}(\mathrm{A})$.

On the other hand

$$
\begin{aligned}
\left(\pi \otimes \operatorname{id}_{\mathrm{A}}\right) & \left(\Delta\left(L_{\omega}(a)\right)\right)=\left(\omega \otimes \pi \otimes \operatorname{id}_{\mathrm{A}}\right)\left(\Delta_{2}(a)\right)=\left(h_{\mathrm{B}} \pi \otimes \pi \otimes \operatorname{id}_{\mathrm{A}}\right)\left(\Delta_{2}(a)\right) \\
= & \left(h_{\mathrm{B}} \otimes \operatorname{id}_{\mathrm{B}} \otimes \operatorname{id}_{\mathrm{A}}\right)\left(\Delta_{\mathrm{B}} \otimes \operatorname{id}_{\mathrm{A}}\right)\left(\pi \otimes \operatorname{id}_{\mathrm{A}}\right)(\Delta(a)) \\
= & \left(h_{\mathrm{B}}(\cdot) 1_{\mathrm{B}} \otimes \operatorname{id}_{\mathrm{A}}\right)\left(\pi \otimes \operatorname{id}_{\mathrm{A}}\right)(\Delta(a))=1_{\mathrm{B}} \otimes L_{\omega}(a),
\end{aligned}
$$

so that if $a \in L_{\omega}(\mathrm{A})$ then $\left(\pi \otimes \operatorname{id}_{\mathrm{A}}\right)(\Delta(a))=1_{\mathrm{B}} \otimes a$.

In Theorem 5.1 of [Sol] Soltan studies the properties of the algebra appearing on the right side of equality (4.1) (he actually works with its right version). In the next theorem we show that the properties established in that theorem do not really depend on the fact that the idempotent state $\omega$ appearing on the left side of (4.1) is a Haar idempotent and provide a simpler proof. 
Theorem 4.2. Let $\mathrm{A}$ be a coamenable locally compact quantum group and let $\mathrm{C}$ be a right invariant $\psi$-expected $C^{*}$-subalgebra of $\mathrm{A}$. Then $\mathrm{C}$ is a nondegenerate $C^{*}$ subalgebra of $\mathrm{A}$. Moreover the map $\alpha:=\left.\Delta\right|_{\mathrm{C}}$ is a nondegenerate ${ }^{*}$-homomorphism from $\mathrm{C}$ to $M(\mathrm{C} \otimes \mathrm{A})$ such that

$$
\begin{gathered}
\left(\operatorname{id}_{\mathrm{C}} \otimes \epsilon\right) \alpha=\mathrm{id}_{\mathrm{C}}, \\
\overline{\operatorname{span}} \alpha(\mathrm{C})\left(1_{\mathrm{C}} \otimes \mathrm{A}\right)=\mathrm{C} \otimes \mathrm{A} .
\end{gathered}
$$

Proof. By Proposition 3.3 the algebra $\mathrm{C}$ is of the form $L_{\omega}(\mathrm{A})$ for some idempotent state $\omega \in A^{*}$. Therefore, by the beginning of the proof of Lemma 2.6, $C$ is a nondegenerate $C^{*}$-subalgebra of $\mathrm{A}$ (and $1_{\mathrm{C}}=1_{\mathrm{A}}$ ). Once the nondegeneracy of $\mathrm{C}$ in A has been established, to show that $\alpha$ is a nondegenerate ${ }^{*}$-homomorphism from C to $M(\mathrm{C} \otimes \mathrm{A})$ it suffices to prove that it takes values in $M(\mathrm{C} \otimes \mathrm{A})$. We need to show that for $c, d \in \mathrm{C}$ and $a \in \mathrm{A}$ the element $x:=\Delta(c)(d \otimes a)$ is in $\mathrm{C} \otimes \mathrm{A}$ (note that we already know that $x$ belongs to $\mathrm{A} \otimes \mathrm{A}$ ). Recall that by coassociativity we have $\left(L_{\omega} \otimes \mathrm{id}_{\mathrm{A}}\right) \Delta=\Delta L_{\omega}$. By Theorem $1.3 L_{\omega}$ is a conditional expectation from A onto $C$. This implies that $L_{\omega} \otimes \mathrm{id}_{\mathrm{A}}$ is also a conditional expectation, this time from $A \otimes A$ onto $C \otimes A$. Use the 'extended' module property of conditional expectations (Lemma 1.4) to observe that

$$
\left(L_{\omega} \otimes \mathrm{id}_{\mathrm{A}}\right)(x)=\left(L_{\omega} \otimes \mathrm{id}_{\mathrm{A}}\right)(\Delta(c))(d \otimes a)=\Delta\left(L_{\omega}(c)\right)(d \otimes a)=\Delta(c)(d \otimes a),
$$

so that $\left(L_{\omega} \otimes \mathrm{id}\right)(x)=x$ and $x \in \mathrm{C} \otimes \mathrm{A}$.

The formula (4.2) is a direct consequence of the defining property of the counit. Property (4.3) can be proved along the lines of the last paragraph - we need to use the fact that for $a, b \in \mathrm{A}$

$$
\left(L_{\omega} \otimes \operatorname{id}_{\mathrm{A}}\right)\left(\Delta(a)\left(1_{\mathrm{A}} \otimes b\right)\right)=\left(L_{\omega} \otimes \operatorname{id}_{\mathrm{A}}\right)(\Delta(a))\left(1_{\mathrm{A}} \otimes b\right) .
$$

Once this is noted, we simply observe that

$$
\begin{aligned}
\overline{\operatorname{span}} \alpha(\mathrm{C})\left(1_{\mathrm{A}} \otimes \mathrm{A}\right) & =\overline{\operatorname{span}}\left\{\Delta\left(L_{\omega}(a)\right)\left(1_{\mathrm{A}} \otimes b\right): a, b \in \mathrm{A}\right\} \\
& =\overline{\operatorname{span}}\left\{\left(L_{\omega} \otimes \operatorname{id}_{\mathrm{A}}\right)\left(\Delta(a)\left(1_{\mathrm{A}} \otimes b\right)\right): a, b \in \mathrm{A}\right\} \\
& =\left(L_{\omega} \otimes \mathrm{id}_{\mathrm{A}}\right)(\mathrm{A} \otimes \mathrm{A})=\mathrm{C} \otimes \mathrm{A} .
\end{aligned}
$$

Using the (right-handed version of the) terminology introduced in Definition 2.6 of $\left[\mathrm{Va}_{2}\right]$ the last theorem can be reformulated in the following way.

Corollary 4.3. If $\mathrm{A}$ is a coamenable locally compact quantum group and $\mathrm{C}$ a right invariant $\psi$-expected $C^{*}$-subalgebra of $\mathrm{A}$, then the coproduct of $\mathrm{A}$ restricts to a continuous coaction of $\mathrm{A}$ on $\mathrm{C}$.

Note finally that the algebra in (4.1) satisfies the assumptions of Theorem 4.2 by Propositions 3.2 and 3.12, so Theorem 4.2 indeed generalizes Theorem 5.1 in [Sot].

Acknowledgments. The work on this paper was started during a special semester on 'Banach algebra and operator space techniques in topological group theory' in Leeds in May/June 2010, funded by the EPSRC grant EP/I002316/1. Both authors would like to thank the organisers of that semester for providing excellent research environment. We also thank Nico Spronk for support which, in particular, enabled AS to visit University of Waterloo in December 2010. AS acknowledges 
useful conversations with Piotr Soltan regarding the last part of the paper. PS was partially supported by Academy of Finland. We thank the referee for helpful comments.

\section{REFERENCES}

[Ba $]$ S. Baaj, Multiplicateurs non bornés, Thése $3^{\text {éme }}$ Cycle, Université Paris VI, 1980.

$\left[\mathrm{Ba}_{2}\right]$ S. Baaj, Représentation réguliere du groupe quantique $E(2)$ de Woronowicz, C. R. Acad. Sci. Paris Sér. I Math. 314 (1992), no. 13, 1021-1026.

[BeT] E. Bédos and L. Tuset, Amenability and co-amenability for locally compact quantum groups, Internat. J. Math. 14 (2003), 865-884.

$\left[\mathrm{FS}_{1}\right]$ U. Franz and A.Skalski, On ergodic properties of convolution operators associated with compact quantum groups, Colloq. Math. 113 (2008), no. 3, 13-23.

[FS $\mathrm{FS}_{2}$ U. Franz and A. Skalski, On idempotent states on quantum groups, J. of Algebra 322 (2009), no. $5,1774-1802$.

[FS 3 U. Franz and A. Skalski, A new characterisation of idempotent states on finite and compact quantum groups, C. R. Math. Acad.Sci.Paris 347 (2009), no.17-18, 991-996, (expanded version available at arXiv:0906.2362).

[FST] U. Franz, A. Skalski and R. Tomatsu, Idempotent states on compact quantum groups and their classification on $U_{q}(2), S U_{q}(2)$, and $S O_{q}(3), J$. Noncomm. Geom., to appear, available at arXiv:0903.2363.

[GKK] W. Groenevelt, E. Koelink and J. Kustermans, The dual quantum group for the quantum group analogue of the normalizer of $S U(1,1)$ in $S L(2, \mathbb{C})$, Int. Math. Res. Not. (2010), no. 7 , $1167-1314$.

[Jac] A. Jacobs, The quantum $E(2)$ group, PhD thesis, K.U. Leuven (2005).

[KaI] Y. Kawada and K. Itô, On the probability distribution on a compact group. I, Proc. Phys.Math. Soc. Japan (3) 22 (1940), 977-998.

$\left[\mathrm{Ku}_{1}\right] \mathrm{J}$. Kustermans, KMS-weights on $C^{*}$-algebras, preprint, available at arXiv:9704008.

$\left[\mathrm{Ku}_{2}\right]$ J. Kustermans, The functional calculus of regular operators on Hilbert $C^{*}$-modules revisited, preprint, available at arXiv:970600\%.

$\left[\mathrm{Ku}_{3}\right]$ J. Kustermans, Locally compact quantum groups in the universal setting, Internat. J. Math. 12 (2001), no. 3, 289-338.

$[\mathrm{KuV}]$ J. Kustermans and S. Vaes, Locally compact quantum groups Ann.Sci.École Norm. Sup. (4) 33 (2000), no. 9, 837-934.

[Lan] E.C. Lance, Hilbert $C^{*}$-modules. A toolkit for operator algebraists, Cambridge University Press, Cambridge, 1995.

[LiS] J.M. Lindsay and A. Skalski, Convolution semigroups of states, Math. Z. 267 (2011), 325339.

[NaW] K. Napiórkowski and S.L. Woronowicz, Operator theory in the $C^{*}$-algebra framework, Rep. Math. Phys. 31 (1992), no. 3, 353-371.

[Pal] A.Pal, A counterexample on idempotent states on a compact quantum group, Lett. Math. Phys. 37 (1996), no. 1, 75-77.

[Pau] V.I. Paulsen, Completely bounded maps and operator algebras, Cambridge University Press, Cambridge, 2002.

[Sa] P. Salmi, Compact quantum subgroups and left invariant $C^{*}$-subalgebras of locally compact quantum groups, J. Funct. Anal. 261 (2011), no. 1, 1-24.

[Soł] P. Sołtan, Examples of non-compact quantum group actions, J. Math. Anal. Appl. 372 (2010), 224-236.

[Tak] M. Takesaki, Theory of operator algebras. II, Springer-Verlag, Berlin, 2003.

[Tom] R. Tomatsu, A characterization of right coideals of quotient type and its application to classification of Poisson boundaries, Comm. Math. Phys. 275 (2007), no. 1, 271-296.

[Va 1 S. Vaes, Locally compact quantum groups, Ph. D. dissertation, Katholieke Universiteit Leuven, Leuven, 2001.

[Va 2 S. Vaes, A new approach to quantum and imprimitivity results, J. Funct. Anal. 229 (2005), 317-374.

[VD] A. Van Daele, The Haar measure on some locally compact quantum groups, preprint of the K.U. Leuven (2000), available at arXiv:math.OA/0109004v1. 
[Wa $\left.{ }_{1}\right]$ S. Wassermann, The slice map problem for $C^{*}$-algebras, Proc. London Math. Soc. (3) 32 (1976), 537-559.

$\left[\mathrm{Wa}_{2}\right]$ S. Wassermann, "Exact $C^{*}-$ Algebras and Related Topics," Seoul National University Research Institute of Mathematics Global Analysis Research Center, Seoul, 1994.

$\left[\mathrm{Wo}_{1}\right]$ S.L. Woronowicz, Unbounded elements affiliated with $C^{*}$-algebras and non-compact quantum groups, Commun. Math. Phys. 136 (1991), 399-432.

[Wo2] S.L. Woronowicz, Quantum E(2)-group and its Pontryagin dual, Lett. Math. Phys. 23 (1991), 251-263.

[Wo3] S.L. Woronowicz, Compact quantum groups, in A. Connes, K. Gawedzki, and J. ZinnJustin, editors, Symétries Quantiques, Les Houches, Session LXIV, 1995, 845-884.

[Wo4] S.L. Woronowicz, Quantum $a z+b$ group on complex plane, Internat. J. Math. 12 (2001), no. $4,461-503$.

[Wo5] S.L. Woronowicz, Haar weight on some quantum groups, Group 24: Physical and mathematical aspects of symmetries, Proceedings of the 24th International Colloquium on Group Theoretical Methods in Physics Paris, 15 - 20 July 2002, pp. 763-772.

[WoZ] S.L. Woronowicz and S. Zakrzewski, Quantum ' $a x+b^{\prime}$ group, Rev. Math. Phys. 14 (2002), no. $7-8,797-828$.

Department of Pure Mathematics, University of Waterloo, Waterloo, On, N2L 3G1, CANAda

E-mail address: pekka.salmi@iki.fi

Institute of Mathematics of the Polish Academy of Sciences, ul.Śniadeckich 8, 00-956 Warszawa, Poland

E-mail address: a.skalski@impan.pl 